\title{
Massive star formation in the GMC G345.5+1.0: spatial distribution of the dust emission ${ }^{\star}$
}

\author{
C. López ${ }^{1}$, L. Bronfman ${ }^{1}$, L.-Å. Nyman ${ }^{2}$, J. May ${ }^{1}$, and G. Garay ${ }^{1}$ \\ 1 Departamento de Astronomía, Universidad de Chile, Casilla 36-D, Santiago, Chile \\ e-mail: clopez@das.uchile.cl \\ 2 European Southern Observatory, Casilla 19001, Santiago, Chile \\ Received 8 October 2010 / Accepted 7 August 2011
}

ABSTRACT

\begin{abstract}
Context. Massive condensations in giant molecular clouds (GMCs) are linked to the formation of high mass stars, which are the principal source of heavy elements and UV radiation, playing an important role in the evolution of galaxies.

Aims. We attemp to make a complete census of massive-star formation within all of GMC G345.5+1.0. This cloud is located one degree above the Galactic plane and at $1.8 \mathrm{kpc}$ from the Sun, thus there is little superposition of dust along the line-of-sight, minimizing confusion effects in identifying individual clumps.

Methods. We observed the $1.2 \mathrm{~mm}$ continuum emission across the whole GMC using the Swedish-ESO Submillimetre Telescope (SEST) Imaging Bolometer Array (SIMBA) mounted on the SEST. Observations have a spatial resolution of $0.2 \mathrm{pc}$ and cover $1.8 \times 2.2$ in the sky with a noise of $20 \mathrm{mJy}^{0}$ beam ${ }^{-1}$.

Results. We identify 201 clumps with diameters between 0.2 and $0.6 \mathrm{pc}$, masses between 3.0 and $1.3 \times 10^{3} M_{\odot}$, and densities between $5 \times 10^{3}$ and $4 \times 10^{5} \mathrm{~cm}^{-3}$. The total mass of the clumps is $1.2 \times 10^{4} M_{\odot}$, thus the efficiency in forming these clumps, estimated as the ratio of the total clump mass to the total GMC mass, is $\sim 0.02$. The clump mass distribution for masses between 10 and $10^{3} M_{\odot}$ is well-fitted by a power law $\mathrm{d} N / \mathrm{d} M \propto M^{-\alpha}$, with a spectral mass index $\alpha$ of $1.7 \pm 0.1$. Given their mass distribution, clumps do not appear to be the direct progenitors of single stars. Comparing the $1.2 \mathrm{~mm}$ continuum emission with infrared images taken by the Midcourse Space Experiment (MSX) and by the Spitzer satellite, we find that at least $20 \%$ of the clumps are forming stars, and at most $\sim 80 \%$ are starless. Six massive-star forming regions (MSFRs) embedded in clumps and associated with IRAS point sources have mean densities of $\sim 10^{5} \mathrm{~cm}^{-3}$, luminosities $>10^{3} L_{\odot}$, and spectral energy distributions that can be modeled with two dust components at different mean temperatures of $28 \pm 5$ and $200 \pm 10 \mathrm{~K}$.
\end{abstract}

Key words. ISM: clouds - stars: formation - dust, extinction - stars: massive - Galaxy: structure - galaxies: star formation

\section{Introduction}

\subsection{Giant molecular clouds}

High-mass stars are known to be born in massive and dense clumps embedded within giant molecular clouds (GMCs; Zinnecker \& Yorke 2007). These GMCs have typical radii of $\sim 60 \mathrm{pc}$, masses of $10^{6} M_{\odot}$ and temperatures of $\sim 10 \mathrm{~K}$, and are found to be concentrated toward the Galactic plane (Grabelsky et al. 1988). This gives rise to spatial and kinematic blending along the line-of-sight. For example, the $\mathrm{CO}$ emission, the most frequently used tracer of molecular gas for GMCs, with a low critical density $\left(\sim 10^{2} \mathrm{~cm}^{-3}\right)$, is often complex with multiple profile components. To overcome these observational difficulties and perform a complete census of massive-star forming regions (MSFRs) in a whole GMC, ideally requires one to study GMCs above and below the Galactic plane using a high density tracer. Recent surveys of dust condensations within whole GMCs have been made toward RCW 106 (Mookerjea et al. 2004), Cygnus X (Motte et al. 2007), and NGC 6334 (Muñoz et al. 2007) using millimeter continuum emission as a high density tracer.

\subsection{Massive-star forming regions}

MSFRs are embedded in GMCs, generating a high Lyman con-

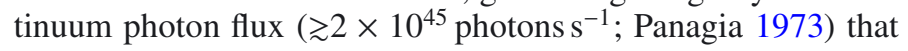

\footnotetext{
* Table 5 is available in electronic form at http://www. aanda.org
}

ionizes the surrounding gas and heats the surrounding dust. These MSFRs can thus be identified by infrared emission from heated dust and/or by radio emission from ionized gas. They can also be identified based on both their molecular lines from high density gas and their millimeter and sub-millimeter continuum emission from dust.

Bronfman et al. (1996) showed that $60 \%$ of the IRAS point sources in the Galactic plane with far infrared (FIR) colors typical of ultra compact (UC) HII regions (Wood \& Churchwell 1989) are associated with dense molecular structures seen in the CS(2-1) line $\left(\gtrsim 10^{4}-10^{5} \mathrm{~cm}^{-3}\right)$. Hereafter, these sources will be referred to as IRAS-CS sources. From observations in $1.2 \mathrm{~mm}$ continuum emission of 146 IRAS-CS sources, Faúndez et al. (2004) showed that MSFRs are associated with condensations of gas and dust. Infrared studies, however, cannot provide a complete census of the birth sites of massive stars, since there are massive condensations that are undetected at infrared wavelengths. For example, Garay et al. (2004) found four clumps with masses between $4 \times 10^{2}$ and $2 \times 10^{3} M_{\odot}$ and densities of $\sim 2 \times 10^{5} \mathrm{~cm}^{-3}$, without infrared emission, located close to clumps associated with MSFRs. They suggested that these cold ( $\lesssim 17 \mathrm{~K})$, dense, and massive clumps will eventually form high mass stars. Hill et al. (2005) found 113 cold clumps, which have a mean mass of $\sim 800 M_{\odot}$, a mean radius of $\sim 0.4 \mathrm{pc}$, and a mean density of $\sim 10^{5} \mathrm{~cm}^{-3}$. Beltrán et al. (2006) found 95 cold clumps with a mean mass of $96 M_{\odot}$, a mean radius of $0.4 \mathrm{pc}$, and a mean density of $9 \times 10^{4} \mathrm{~cm}^{-3}$. 


\subsection{Clumps in GMCs}

Observations in molecular lines (e.g. Bains et al. 2006) and dust continuum emission (e.g. Muñoz et al. 2007) on spatial scales smaller than $\sim 1 \mathrm{pc}$ show that GMCs have a fragmented structure, and these sub-structures have been referred to as clumps (Williams et al. 2000).

Clumps in GMCs have masses from 4 to $10^{4} M_{\odot}$, diameters from 0.2 to $2 \mathrm{pc}$, and densities from $10^{3}$ to $10^{5} \mathrm{~cm}^{-3}$. The clump mass distribution is consistent with a power law $\mathrm{d} N / \mathrm{d} M \propto M^{-\alpha}$, where $\mathrm{d} N / \mathrm{d} M$ is the number of objects by mass interval, $M$ is the mass, and $\alpha$ is the mass spectral index. The derived mass spectral indices range between 1.3 and 1.8 (e.g. Mookerjea et al. 2004; Muñoz et al. 2007), values similar to those found for the mass distribution of molecular clouds as a whole $(\alpha=1.5-1.6$; Sanders et al. 1985; Solomon et al. 1987; Williams \& McKee 1997). The similarity between the spectral mass indices suggests a common origin; however, different mechanisms have been proposed to explain the formation of clumps and GMCs. On the one hand, large-scale gravitational instabilities, in the combined medium of the collisionless stars and the collisional gas, drive spiral density waves (e.g. Li et al. 2005), and are likely to be the main mechanism behind GMC formation. On the other hand, since GMCs are considered turbulent and supersonic, it is expected that the clump formation is, to first order, produced by ram pressures from supersonic flows, which can provide the seeds for a gravitational fragmentation (BallesterosParedes et al. 2006, and 2007; Bonnell et al. 2007; Klessen et al. 1998).

\subsection{This paper}

To undertake a complete census of dense and massive clumps, including those with and without infrared emission, we observed the whole of GMC G345.5+1.0 in $1.2 \mathrm{~mm}$ continuum emission. The determination of physical properties of a sample of clumps belonging to a single GMC is desirable since, in this way, the distance to the GMC is a factor that influences neither the mass distribution or the relationships between physical properties. However, these studies are difficult because of the superposition of dust and molecular structures along the lineof-sight to GMCs in the Galactic plane. We chose the GMC $\mathrm{G} 345.5+1.0$ as our target for two reasons. First, it is located $\sim 1^{\circ}$ above the Galactic plane, so our observations in $1.2 \mathrm{~mm}$ continuum emission are roughly free of confusion with background or foreground structures along the line-of-sight. Second, it is at $1.8 \mathrm{kpc}$ from the Sun, near enough to resolve clumps associated with MSFRs, but far enough away to permit a complete coverage of the GMC.

The structure of this article is as follows: Sects. 2 and 3 present, respectively, the main characteristics of G345.5+1.0 and the observations; Sect. 4 presents the results and a discussion, and Sect. 5 gives a summary of our study.

\section{GMC G345.5+1.0}

The GMC G345.5+1.0 was first observed as part of the Columbia University - Universidad de Chile ${ }^{12} \mathrm{CO}(1-0)$ Survey of the Southern Galaxy (Bronfman et al. 1989). It is located approximately between $344^{\circ} .5$ and 346.5 in Galactic longitude, and between 0.2 and 2.0 in Galactic latitude. Figure 1 shows the spectrum of the ${ }^{12} \mathrm{CO}(1-0)$ line emission integrated over the area of the GMC. The emission of the GMC is between -33 and $-2 \mathrm{~km} \mathrm{~s}^{-1}$ (LSR velocities) with a peak at $-13.6 \mathrm{~km} \mathrm{~s}^{-1}$.

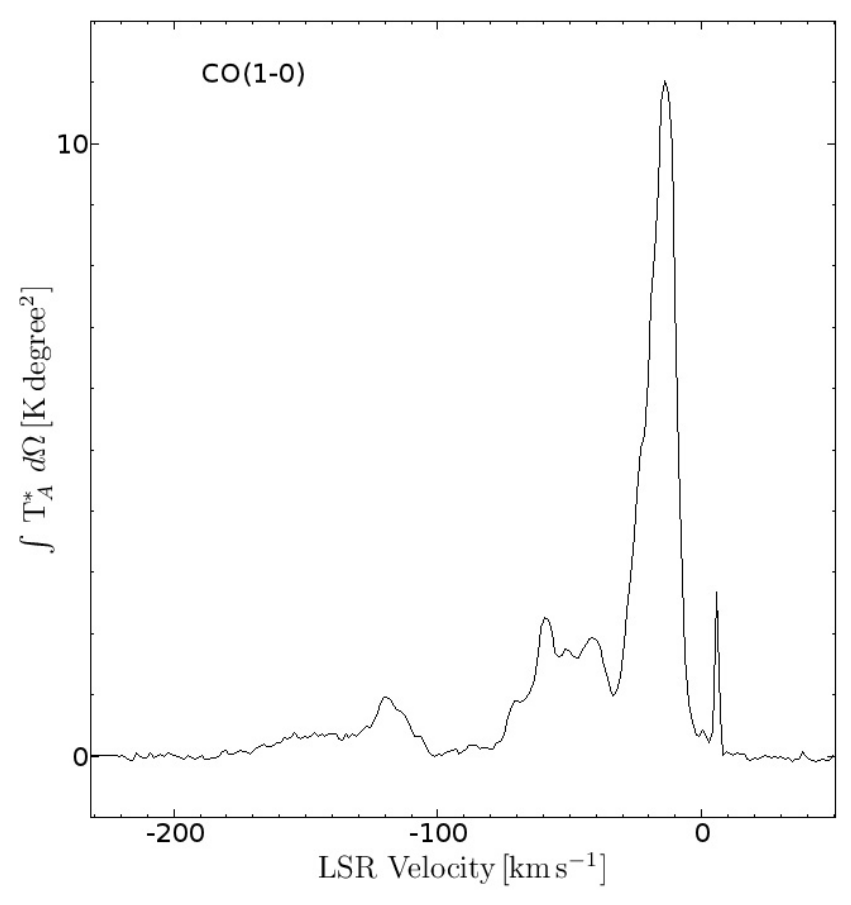

Fig. 1. Spectra of the ${ }^{12} \mathrm{CO}(1-0)$ line emission integrated over the whole area of the GMC G345.5+1.0 (between 344.5 and 346.5 in Galactic longitude and between 0.2 and 2.0 in Galactic latitude; Bronfman et al. 1989). Emission from the GMC under study is between -33 and $-2 \mathrm{~km} \mathrm{~s}^{-1}$ with a peak at $-13.6 \mathrm{~km} \mathrm{~s}^{-1}$.

We estimate the kinematic distance using the rotation curve determined by Alvarez et al. (1990), with a Galactocentric solar distance of $8.5 \mathrm{kpc}$ and a solar LSR velocity of $220 \mathrm{~km} \mathrm{~s}^{-1}$. Considering a Galactic longitude of $345^{\circ} .5$ and LSR velocity of $-13.6 \mathrm{~km} \mathrm{~s}^{-1}$, the GMC is within the solar circle with two possible kinematic distances: $\sim 1.8$ and $15 \mathrm{kpc}$. Thus, the GMC is $\sim 31$ or 262 pc above the Galactic plane, a factor 0.5 or 4.4 of the HWHM of the molecular Galactic disk ( $\sim 60 \mathrm{pc}$; Bronfman et al. 2000), respectively. Therefore, $1.8 \mathrm{kpc}$ is the most probable kinematic distance to the GMC.

Physical properties of the GMC are estimated using the ${ }^{12} \mathrm{CO}(1-0)$ line observations. The ${ }^{12} \mathrm{CO}(1-0)$ line emission integrated over the full spatial and spectral extension of the GMC is $192 \mathrm{~K} \mathrm{~km} \mathrm{~s}^{-1} \mathrm{deg}^{2}$. Using a ratio of $\mathrm{H}_{2}$ column density to integrated ${ }^{12} \mathrm{CO}(1-0)$ line emission $N_{\mathrm{H}_{2}} / W_{\text {co }}$ equal to $1.56 \times 10^{20} \mathrm{~cm}^{-2}\left(\mathrm{~K} \mathrm{~km} \mathrm{~s}^{-1}\right)^{-1}$ (Hunter et al. 1997), the total mass of the GMC is $6.3 \times 10^{5} M_{\odot}$, corrected by a factor of 1.3 to account for $25 \%$ of helium. Since its angular size is $2.0 \times 1.8$, the GMC has a mean radius of $\sim 34 \mathrm{pc}$, mean column density of $\sim 10^{22} \mathrm{~cm}^{-2}$ and mean density of $\sim 70 \mathrm{~cm}^{-3}$, where the depth of the cloud has been assumed to be same as its radius. Table 1 summarises the main characteristics of this GMC. The derived physical properties confirm that the most probable distance to the GMC is $\sim 1.8 \mathrm{kpc}$. If at the far kinematic distance $(15 \mathrm{kpc})$, the GMC would have a mean radius of $280 \mathrm{pc}$ and a total mass of $4.4 \times 10^{7} M_{\odot}$, values much higher than the typical value of $60 \mathrm{pc}$ in radius and $10^{6} M_{\odot}$ in mass (Dame et al. 1986); Williams \& McKee (1997) found that the molecular cloud mass distribution within the solar circle has an upper mass limit of $6 \times 10^{6} M_{\odot}$.

\section{Observations}

We observed the whole GMC G345.5+1.0 in continuum emission at $1.2 \mathrm{~mm}$ using SIMBA mounted on the SEST. The SEST 
Table 1. Summary of main characteristics of G345.5+1.0.

\begin{tabular}{lc}
\hline \hline Distance & $1.8 \mathrm{kpc}$ \\
Total mass & $6.5 \times 10^{5} M_{\odot}$ \\
Radius & $34 \mathrm{pc}$ \\
${ }^{a}$ Density & $70 \mathrm{~cm}^{-3}$ \\
${ }^{a}$ Column density & $10^{22} \mathrm{~cm}^{-2}$ \\
LSR velocity & $-13.6 \mathrm{~km} \mathrm{~s}^{-1}$ (between -33 and $-2 \mathrm{~km} \mathrm{~s}^{-1}$ ) \\
\hline
\end{tabular}

Notes. ${ }^{(a)}$ The density and the column density are computed assuming a mean molecular weight of $\mu=2.29$.

is a $15-\mathrm{m}$ diameter radio telescope, which operated between 70 and $365 \mathrm{GHz}$, and SIMBA is a 37-channel hexagonal bolometer, operating at $250 \mathrm{GHz}(1.2 \mathrm{~mm})$, with a passband equivalent width of $90 \mathrm{GHz}$ (FWHM). The configuration SEST-SIMBA had a beamsize of $24^{\prime \prime}$, which corresponds to a spatial resolution of $\sim 0.2 \mathrm{pc}$ at the GMC distance $(1.8 \mathrm{kpc})$.

Observations were made in October 2002 and July 2003, using the fast mapping mode, and consist of 185 images of $15^{\prime}$ (azimuth) $\times 10^{\prime}$ (elevation) in size. The scans were made in azimuth at a rate of $80^{\prime \prime} \mathrm{s}^{-1}$, and they were separated in elevation by $8^{\prime \prime}$; the total integration time per map was about $25 \mathrm{~min}$. Measurements of the atmospheric opacity were made through skydips about every three hours, and values at the zenith ranged between 0.09 and 0.31. Data were reduced using the MOPSI software (developed by Robert Zylka, IRAM, Grenoble, France), and calibrated (in terms of flux density) with observations toward Uranus, one made in October 2002, and an additional eight made in July 2003. Bolometer channels were corrected for the correlated noise by inspecting the surrounding channels. The noise correlation between 1 and 900 arcsec, and between 100 and 900 arcsec inferred an error smaller than $20 \%$ in the flux densities of detected sources. The calibration factor has a value of $0.086 \mathrm{Jy}_{\text {counts }}{ }^{-1}$ for October 2002, and a mean value of $\sim 0.069 \pm 0.005 \mathrm{Jy} \mathrm{counts}^{-1}$ for July 2003 . These variations agree with the uncertainty estimated by Faúndez et al. (2004) of $20 \%$ in the flux density measurements using SIMBA data.

For the 185 images, we achieved an rms of between 0.023 and $0.080 \mathrm{Jy} \mathrm{beam}^{-1}$, with a median of $0.036 \mathrm{Jy} \mathrm{beam}^{-1}$, for a beam calibration area of $\sim 653 \operatorname{arcsec}^{2}$ ("SIMBA Data Reduction Handbook"). The individual maps were combined in a mosaic of $1.8 \times 2.2$ in size, centered at $345^{\circ} .40$ in Galactic longitude and +1.10 in Galactic latitude, and with a final rms of $\sim 20 \mathrm{mJy}^{\text {beam }^{-1}}$.

\section{Results and discussion}

\subsection{The $1.2 \mathrm{~mm}$ continuum emission}

Figure 2 presents the $1.2 \mathrm{~mm}$ emission image of the whole GMC G345.5+1.0. The total flux density of the GMC G345.5+1.0 is $\sim 365 \mathrm{Jy}$, which is estimated by integrating the intensity over the whole area of the cloud.

As can be seen in the integrated spectrum of the ${ }^{12} \mathrm{CO}(1-0)$ line (Fig. 1), across GMC G345.5+1.0 (LSR velocity between -33 and $-2 \mathrm{~km} \mathrm{~s}^{-1}$ ), there are additional molecular gas components along the line-of-sight, particularly in the ranges -170 to $-100 \mathrm{~km} \mathrm{~s}^{-1},-75$ to $-33 \mathrm{~km} \mathrm{~s}^{-1}$, and 0 to $5 \mathrm{~km} \mathrm{~s}^{-1}$. Hence, the question arises as to whether the $1.2 \mathrm{~mm}$ continuum emission only traces dust condensations within GMC G345.5+1.0? To examine the association of the GMC with
Table 2. List of IRAS point sources along the line-of-sight to the GMC G345.5+1.0 observed in the CS(2-1) line by Bronfman et al. (1996).

\begin{tabular}{ccccc}
\hline \hline IRAS name & \multicolumn{2}{c}{ Galactic coord. } & LSR velocity $^{1}$ & $F W H M^{1}$ \\
& longitude & latitude & [ $\left.\mathrm{km} \mathrm{s}^{-1}\right]$ & {$\left[\mathrm{km} \mathrm{s}^{-1}\right]$} \\
\hline $16533-4022$ & 344.845 & 1.646 & undetected & \\
$16571-4029$ & 345.208 & 1.028 & -15.6 & 4.9 \\
$16577-4028$ & 345.286 & 0.933 & undetected & \\
$16575-4023$ & 345.332 & 1.014 & -14.5 & 2.8 \\
$16561-4006$ & 345.393 & 1.399 & -11.9 & 3.5 \\
$16557-4002$ & 345.395 & 1.512 & -12.4 & 3.1 \\
$17009-4042$ & 345.490 & 0.311 & -16.7 & 6.0 \\
$16562-3959$ & 345.494 & 1.468 & -11.6 & 5.5 \\
$17008-4040$ & 345.499 & 0.354 & -16.4 & 4.8 \\
$16596-4012$ & 345.717 & 0.817 & -11.5 & 4.9 \\
\hline
\end{tabular}

Notes. ${ }^{(1)}$ Values from Bronfman et al. (1996).

$1.2 \mathrm{~mm}$ continuum emission, Fig. 3 shows images of the velocity-integrated ${ }^{12} \mathrm{CO}(1-0)$ emission in three velocity ranges ( -200 to $-33 \mathrm{~km} \mathrm{~s}^{-1},-33$ to $-2 \mathrm{~km} \mathrm{~s}^{-1}$, and -2 to $50 \mathrm{~km} \mathrm{~s}^{-1}$ ) superimposed with contours of the $1.2 \mathrm{~mm}$ continuum emission. Figure 3 shows that most of the emission detected in $1.2 \mathrm{~mm}$ is associated with the GMC. About $1 \%$ of the total observed area might also be associated with gas at velocities $<-33 \mathrm{~km} \mathrm{~s}^{-1}$ (Fig. 3, top), localized mainly in the region of the IRAS point sources 17008-4040 (G345.499+0.354) and 170094042 (G345.490+0.311).

From the survey of Bronfman et al. (1996), we find that there are eight IRAS-CS sources within the region and two IRAS point sources that are not detected in the $\mathrm{CS}(2-1)$ line (see Table 2). As is shown in both the ${ }^{12} \mathrm{CO}(1-0)$ maps (Fig. 3) and the $1.2 \mathrm{~mm}$ continuum emission map (Fig. 4), these MSFRs, or IRAS-CS sources, are associated with the GMC and have a counterpart in $1.2 \mathrm{~mm}$. They correspond to the most dense and massive dust condensations (see Table 5). The two IRAS point sources not detected in the $\mathrm{CS}(2-1)$ line were also not detected in the continuum (see Fig. 4). The eight IRAS-CS sources include the IRAS point sources 17008-4040 and 17009-4042. Line profiles toward these two objects in the ${ }^{12} \mathrm{CO}(1-0)$ and $\mathrm{CS}(2-1)$ lines are shown in Fig. 5. Gas components with velocities $<-33 \mathrm{~km} \mathrm{~s}^{-1}$ observed in the ${ }^{12} \mathrm{CO}(1-0)$ line are not observed in the $\mathrm{CS}(2-1)$ line, suggesting that they correspond to regions of low density gas. Since $1.2 \mathrm{~mm}$ continuum emission traces high densities (e.g. Faúndez et al. 2004), it should not be detected in these clouds. In summary, from observations in the ${ }^{12} \mathrm{CO}(1-0)$ and $\mathrm{CS}(2-1)$ lines, we conclude that the $1.2 \mathrm{~mm}$ continuum emission is associated only with the GMC.

\subsection{Identification of clumps}

The structure of the GMC observed in $1.2 \mathrm{~mm}$ continuum emission (Fig. 2) is fragmented, and it is possible to distinguish several clumps.

To identify clumps we utilize CLUMPFIND ${ }^{1}$ (Williams et al. 1994), which creates contours over data, searches for peaks of emission to locate clumps, and follows them down to the lower intensity contour.

CLUMPFIND finds 201 clumps in the $1.2 \mathrm{~mm}$ continuum emission map of the GMC, containing $\sim 100 \%$ of the total emission above $3 \sigma$. We used a lower intensity contour of three rms, $\sim 0.06 \mathrm{Jy} \mathrm{beam}^{-1}$, and a contouring interval equal to twice

${ }^{1}$ http://www.ifa.hawaii.edu/users/jpw/clumpfind.shtml 


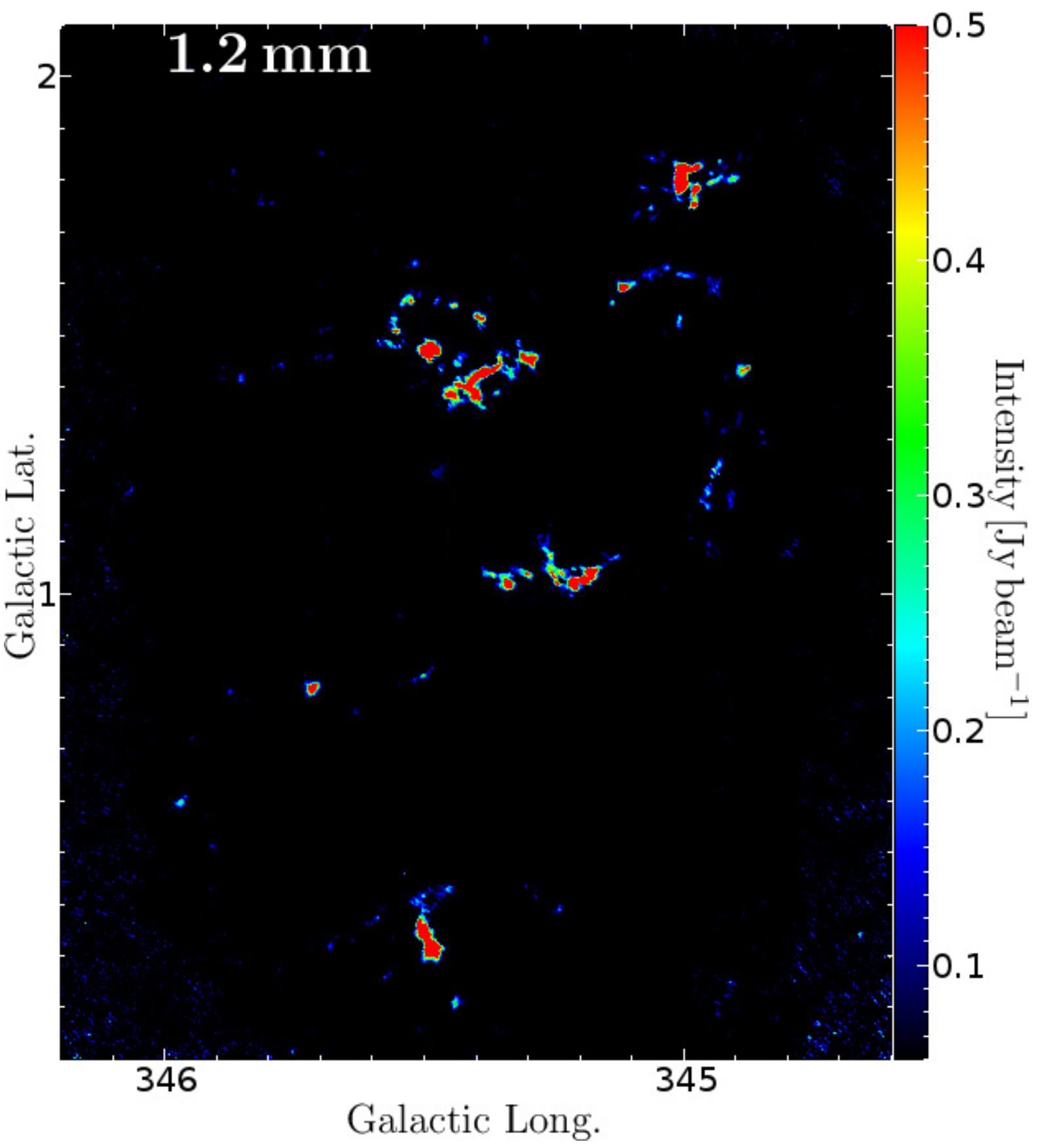

Fig. 2. Map of the GMC G345.5+1.0 in $1.2 \mathrm{~mm}$ continuum emission. Observations were made using SIMBA, with a spatial resolution of 0.2 pc. They cover $1.8 \times 2.2$ in the sky, with an rms of $20 \mathrm{mJy}^{1}$ beam $^{-1}$.

the rms noise, $\sim 0.04 \mathrm{Jy}_{\text {beam }}{ }^{-1}$. To delete fictitious structures, we imposed two conditions on the CLUMPFIND output, that the angular size of the emission and the emission peak of clumps had to be greater than the beam size, $\sim 24^{\prime \prime} \times 24^{\prime \prime}$, and five times rms, $\sim 0.1 \mathrm{Jy}_{\text {beam }}^{-1}$, respectively. The angular size is defined to be the angular area inside the lowest intensity contour (three rms). The 201 identified clumps have areas between $\sim 0.18$ and $7.3 \mathrm{arcmin}^{2}$, emission peaks between 0.1 and $9 \mathrm{Jy} \mathrm{beam}^{-1}$, and flux densities between 0.089 and $40 \mathrm{Jy}$.

Table 5 shows the characteristics of each clump calculated in this section, Sects. 4.3 and 4.4. Column 1 gives clump names; Cols. 2 and 3, Galactic coordinates of peaks in $1.2 \mathrm{~mm}$ continuum emission; Col. 4, $1.2 \mathrm{~mm}$ flux densities; Col. 5, diameters (deconvolved FWHM sizes); Col. 6, masses; Col. 7, densities; Col. 8, column densities; and Col. 9, if clumps have ("Y") or do not have ("N") an infrared counterpart from MSX and Spitzer observations.

\subsection{Physical properties of clumps}

First, we estimate the minimum gas column density that can be detected given the rms noise of our observations. Assuming that $1.2 \mathrm{~mm}$ continuum emission is optically thin and produced by dust, the column density $N$ is (Hildebrand 1983)

$N=\frac{S_{1.2 \mathrm{~mm}} R_{\mathrm{gd}}}{\Omega \mu m_{\mathrm{H}} k_{1.2 \mathrm{~mm}} B_{1.2 \mathrm{~mm}}\left(T_{\text {dust }}\right)}$,

where $\Omega$ is the beam solid angle, $S_{1.2 \mathrm{~mm}}$ is the flux density at $1.2 \mathrm{~mm}, \mu$ is the mean mass per particle, equal to $\sim 2.29$ for an $\mathrm{H}_{2}$ cloud with a $25 \%$ contribution of helium (Evans 1999), $m_{\mathrm{H}}$ 

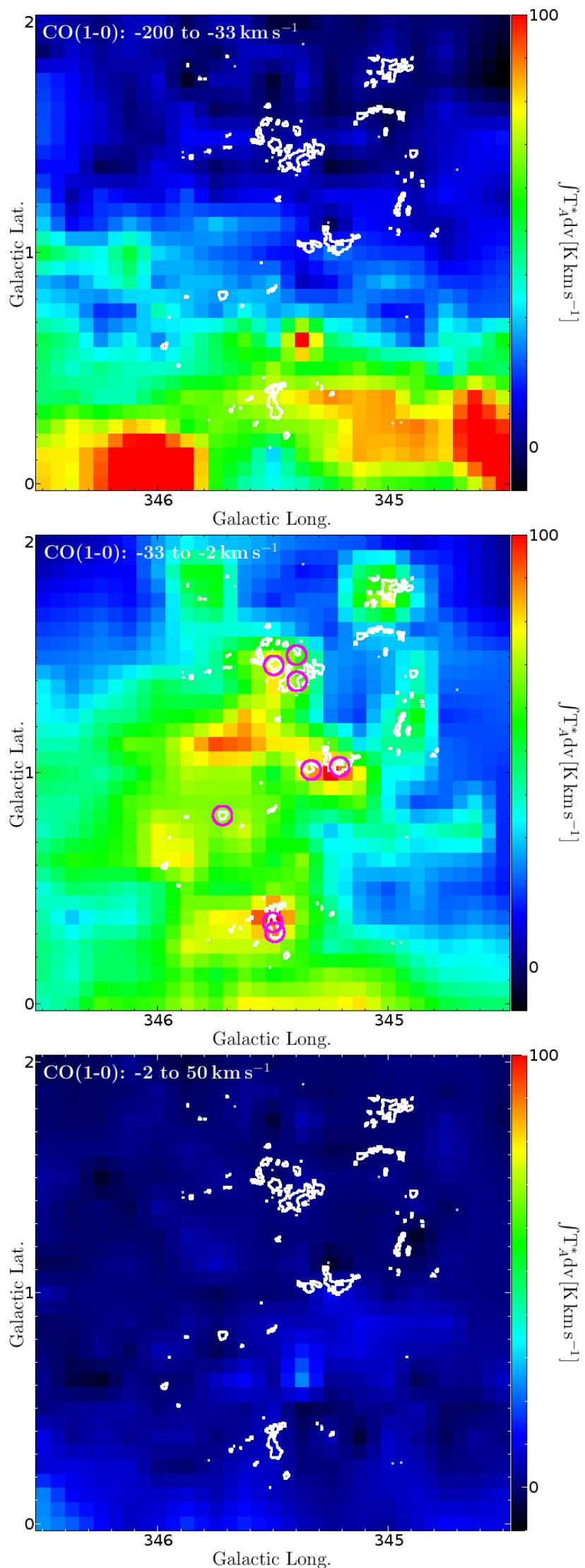

Fig. 3. Integrated ${ }^{12} \mathrm{CO}(1-0)$ emission toward GMC G345.5+1.0 in different LSR velocity ranges (Bronfman et al. 1989). Top: from -200 to $-33 \mathrm{~km} \mathrm{~s}^{-1}$. Middle: from -33 to $-2 \mathrm{~km} \mathrm{~s}^{-1}$. Bottom: from -2 to $50 \mathrm{~km} \mathrm{~s}^{-1}$. Magenta circles mark spatial and spectral positions of detections in the CS(2-1) line toward MSFRs (Table 2). Contours represent $1.2 \mathrm{~mm}$ continuum emission at 5 times rms, $\sim 0.1 \mathrm{Jy}^{\text {beam }}{ }^{-1}$.

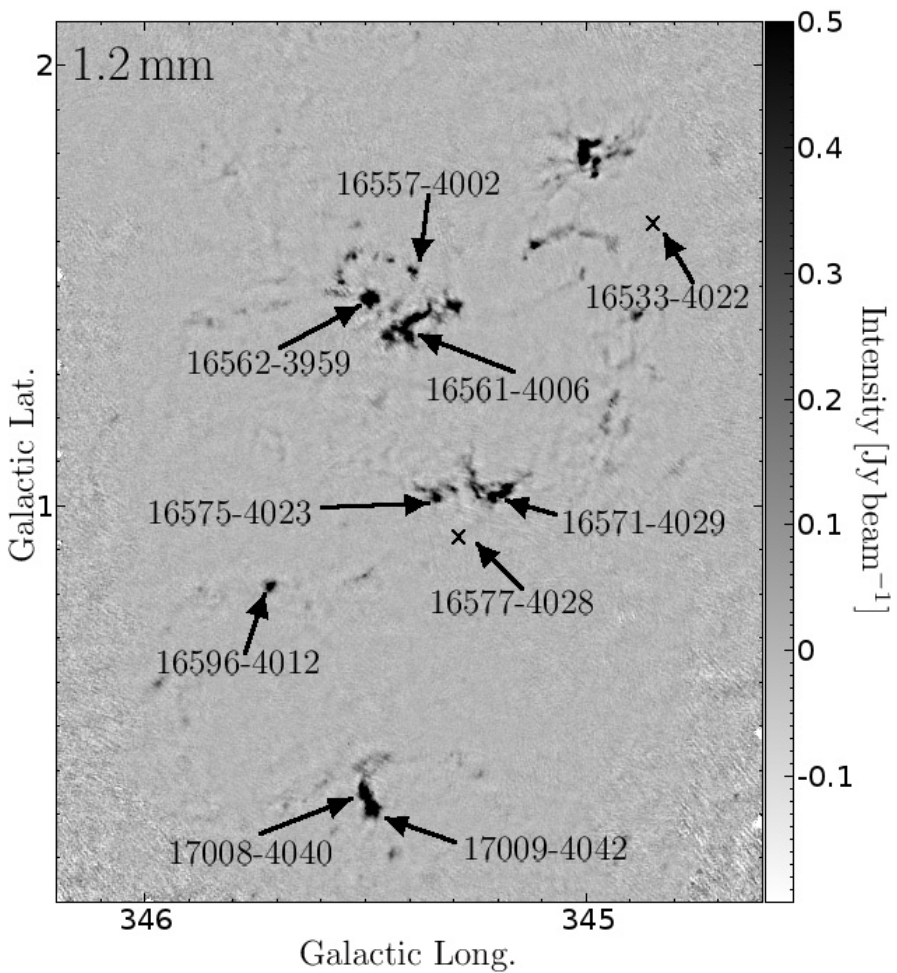

Fig. 4. IRAS point sources along the line-of-sight of the GMC G345.5+1.0 observed in the CS(2-1) line (Bronfman et al. 1996). Gray scale represents $1.2 \mathrm{~mm}$ continuum emission. Arrows mark CS(2-1) line observations, and crosses indicate observations without detection (see Table 2).

is the hydrogen atom mass, $k_{1.2 \mathrm{~mm}}$ is the dust absorption coefficient at $1.2 \mathrm{~mm}$, equal to $\sim 1 \mathrm{~cm}^{2} \mathrm{~g}^{-1}$ for protostellar cores (Ossenkopf \& Henning 1994), $B_{1.2 \mathrm{~mm}}\left(T_{\text {dust }}\right)$ is the Planck function at both $1.2 \mathrm{~mm}$ and a dust temperature $T_{\text {dust }}$, equal to $\sim 30 \mathrm{~K}$ for regions of massive-star formation (Faúndez et al. 2004), and $R_{\mathrm{gd}}$ is the ratio of gas to dust mass, $\sim 100$ (Hildebrand 1983). For a solid angle limit of $24^{\prime \prime} \times 24^{\prime \prime}$ and an intensity limit of five rms, $\sim 0.1 \mathrm{Jy}$ beam $^{-1}$, the minimum flux density is $\sim 88 \mathrm{mJy}$ and the minimum column density that can be detected is $\sim 4 \times 10^{21} \mathrm{~cm}^{-2}$, which corresponds to a visual extinction of $4 \mathrm{mag}$, assuming that $N_{\mathrm{H}_{2}} / A_{\mathrm{V}} \sim 10^{21} \mathrm{~cm}^{-2} \mathrm{mag}^{-1}$ (Bohlin et al. 1978).

Masses of clumps, $M_{\mathrm{c}}$, are estimated as

$M_{\mathrm{c}}=\int \mu m_{\mathrm{H}} N \mathrm{~d} A=\frac{S_{1.2 \mathrm{~mm}} R_{\mathrm{gd}} d^{2}}{B_{1.2 \mathrm{~mm}}\left(T_{\text {dust }}\right) k_{1.2 \mathrm{~mm}}}$,

where $\mathrm{d} A$ is the differential element of area $\left(\mathrm{d} A=\mathrm{d}^{2} \mathrm{~d} \Omega\right)$ and $d$ is the distance to the GMC $(\sim 1.8 \mathrm{kpc})$. Since we insist that identified clumps have intensities and dimensions greater than $0.1 \mathrm{Jy} \mathrm{beam}^{-1}$ and $24^{\prime \prime} \times 24^{\prime \prime}$ respectively, the lower limit to their masses is $\sim 2.9 M_{\odot}$. The derived masses of clumps range from 3.0 to $1.3 \times 10^{3} M_{\odot}$, with a total mass of $1.2 \times 10^{4} M_{\odot}$ (see Tables 3 and 5). The efficiency in forming these clumps, estimated as the ratio of the total clump mass to the total GMC mass, is thus $\sim 0.02$.

The clump mass distribution (CMD) is shown in Fig. 6, plotted as $\mathrm{d} N / \mathrm{d} \log \left(M / M_{\odot}\right)$ versus mass, where $\mathrm{d} N / \mathrm{d} \log \left(M / M_{\odot}\right)$ is approximated by the number of clumps, $\Delta N$, within a logarithmic mass interval $\Delta \log \left(M / M_{\odot}\right)$. In this figure, $\Delta \log \left(M / M_{\odot}\right)$ is constant, at a value of $\sim 0.44$. The CMD is well-fitted by a power law $\mathrm{d} N / \mathrm{d} \log (M) \propto M^{-\alpha+1}$, which can be expressed as $\mathrm{d} N / \mathrm{d} M \propto$ $M^{-\alpha}$, with the spectral mass index, $\alpha$, equal to $1.7 \pm 0.1$ for 

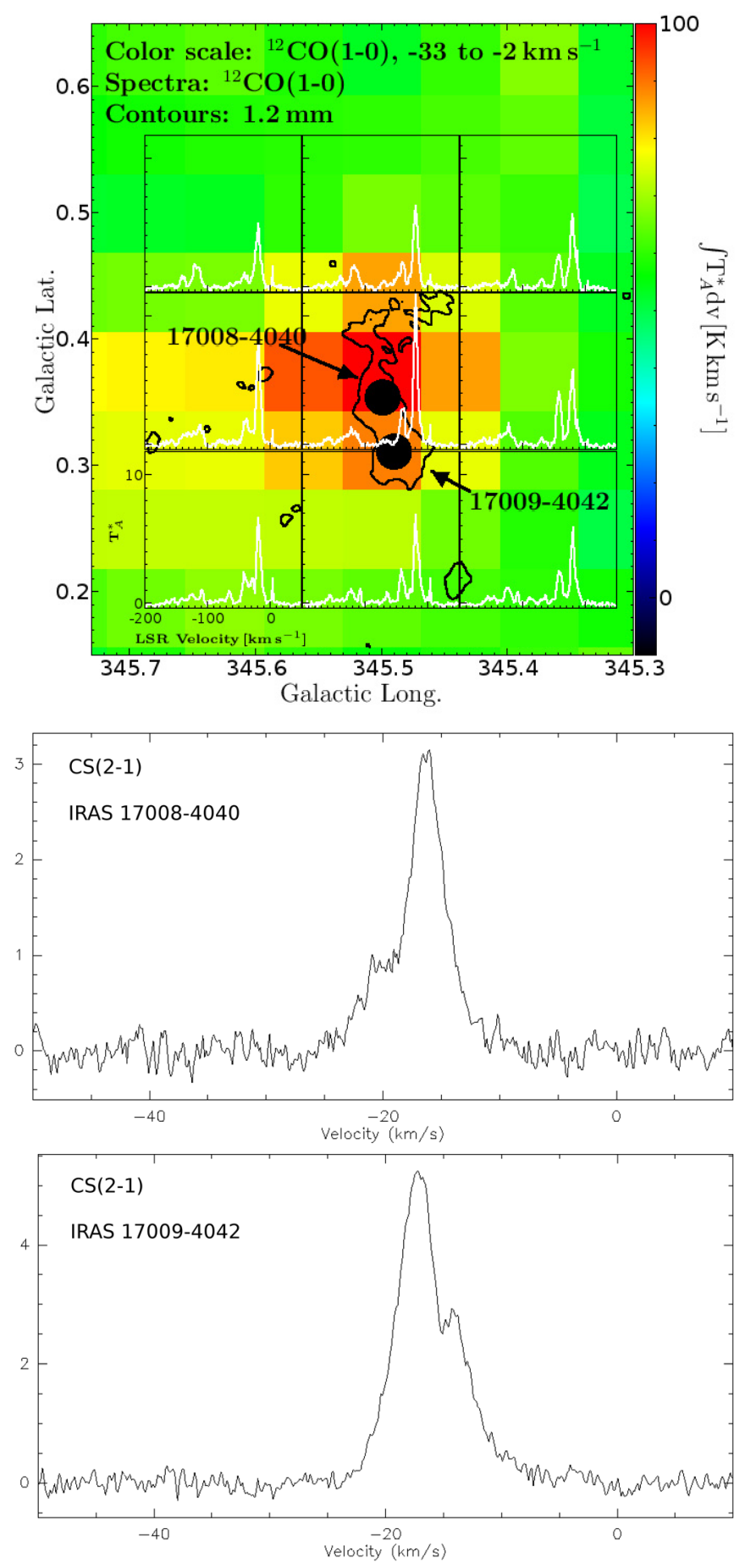

Fig. 5. Line profiles toward the IRAS point sources $17008-4040$ and 17009-4042: top image, ${ }^{12} \mathrm{CO}(1-0)$ line profiles (Bronfman et al. 1989), over a map of their integrated emissions (color scale) and with contours of $1.2 \mathrm{~mm}$ continuum emission; middle and bottom images, CS(2-1) line profiles (their observing positions are indicated as black dots in the top image; Bronfman et al. 1996).

masses between $\sim 10$ and $1.3 \times 10^{3} M_{\odot}$. The correlation coefficient of the fit is 0.993 . Between $10 M_{\odot}$ and $1.3 \times 10^{3} M_{\odot}$, bin size variations of $\Delta \log \left(M / M_{\odot}\right)$ between 0.18 and 1.1 result in values of $\alpha$ consistent with $1.7 \pm 0.1$. Since $\alpha$ is 1.7 , the population is dominated by clumps with low masses, but the total mass is dominated by the most massive clumps; for example, $50 \%$ of the population is between 10 and $27 M_{\odot}$, but contains only $10 \%$ of the total mass. The turnover below $\sim 10 M_{\odot}$ is produced by

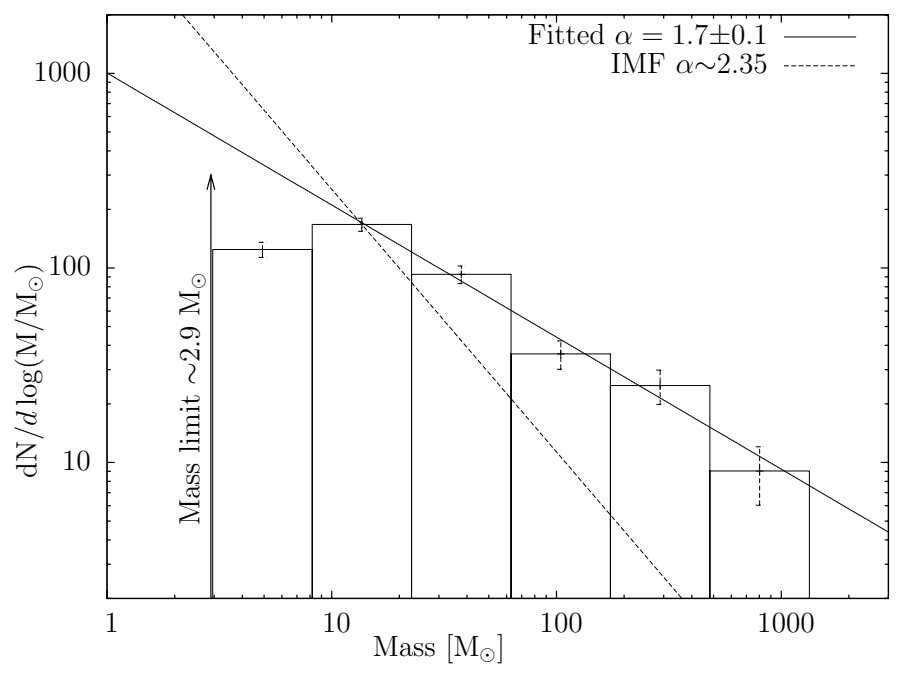

Fig. 6. Mass distribution of identified clumps in G345.5+1.0, plotted as $\mathrm{d} N / \mathrm{d} \log \left(M / M_{\odot}\right)$ versus mass, where $\mathrm{d} N / \mathrm{d} \log \left(M / M_{\odot}\right)$ is approximated by the number of clumps $\Delta N$ within a logarithmic mass interval $\Delta \log \left(M / M_{\odot}\right)$. Here, $\Delta \log \left(M / M_{\odot}\right)$ is constant, $\sim 0.44$. Error bars are estimated by $\sqrt{\Delta N / \Delta \log \left(M / M_{\odot}\right)}$. The arrow shows the clump mass limit, $\sim 2.9 M_{\odot}$. The continuous line represents the mass distribution fit with $\mathrm{d} N / \operatorname{d} \log \left(M / M_{\odot}\right) \propto M^{1-\alpha}$, where the spectral mass index $\alpha$ is $1.7 \pm 0.1$ for masses between $\sim 10$ and $1.3 \times 10^{3} M_{\odot}$. The dashed line displays the spectral mass index for the stellar initial mass function (IMF) of the solar neighborhood for stellar masses greater than $0.5 M_{\odot}$ (e.g. Kroupa 2002); the line is forced to pass through the peak of the clump mass distribution.

an incompleteness of the clump catalog caused by the combination of the spatial resolution and flux density limit of the survey. However, observations of higher spatial resolution $(\lessgtr 0.01 \mathrm{pc})$ could result in a spectral mass index of $\sim 2.35$, resolving core structures (e.g. Motte et al. 1998). Beltrán et al. (2006) studied a sample of IRAS sources associated with MSFRs in $1.2 \mathrm{~mm}$ continuum and found a spectral mass index of 1.5 for clumps with masses between $\sim 10 M_{\odot}$ and $10^{2} M_{\odot}$ and 2.1 for clumps with masses between $\sim 10^{2}$ and $10^{4} M_{\odot}$. For clumps identified here with masses higher than $100 M_{\odot}$, we find a spectral mass index of $1.6 \pm 0.1$, as is shown in Fig. 7 , in agreement with the previous fit considering clumps with masses between 10 and $1.3 \times 10^{3} M_{\odot}$. It is necessary to observe more whole GMCs to confirm these results.

Clump diameters, $D_{\mathrm{c}}$, are estimated from the deconvolved $F W H M$ size of their emissions. We used the FWHM size $\theta_{F W H M}$ estimated by CLUMPFIND algorithm, thus

$D_{\mathrm{c}}=\sqrt{\theta_{F W H M}^{2}-\theta_{\text {beam }}^{2}}$

where $\theta_{\text {beam }}$ is the beam-size. Considering a distance of $1.8 \mathrm{kpc}$ to the GMC and clumps that have a reliable $D_{\mathrm{c}}$, i.e. $D_{\mathrm{c}} \geq \theta_{\text {beam }}$, clumps have diameters between 0.2 and $0.6 \mathrm{pc}$.

From the masses and sizes, and assuming a spherical and homogeneous density distribution, we estimate mean clump densities, using the expression

$\rho=\mu m_{\mathrm{H}} n$,

where $\rho$ is the mass density and $n$ is the particle density. Densities of clumps are between $5 \times 10^{3}$ and $4 \times 10^{5} \mathrm{~cm}^{-3}$. Mean column densities of clumps, $N_{\mathrm{c}}$, are estimated as

$N_{\mathrm{c}} \sim \frac{M_{\mathrm{c}}}{\mu m_{\mathrm{H}} \pi\left(D_{\mathrm{c}} / 2\right)^{2}}$, 


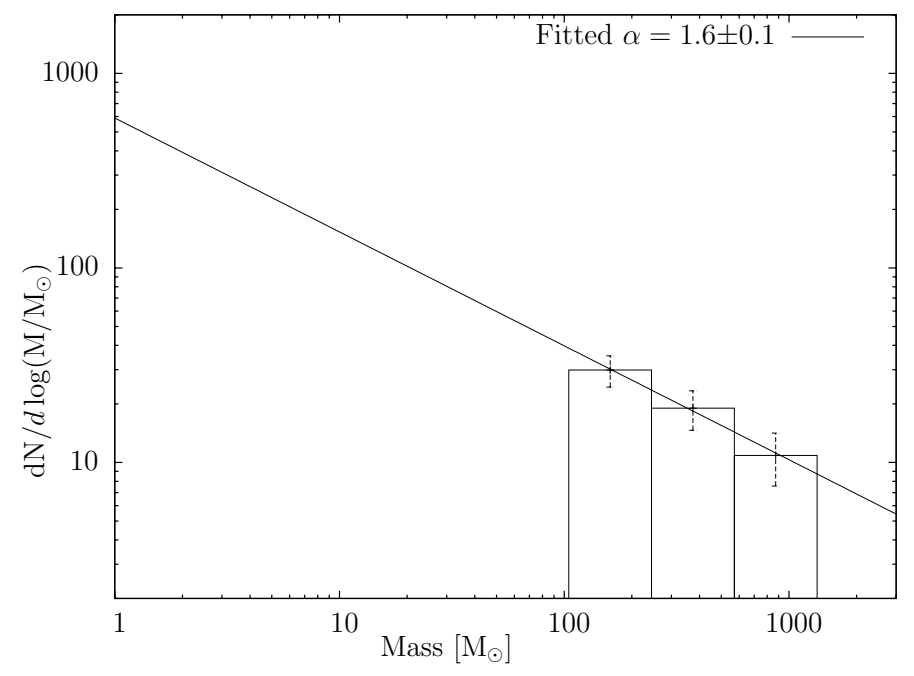

Fig. 7. Mass distribution of identified clumps in G345.5+1.0 with masses higher than $100 M_{\odot}$, plotted as $\mathrm{d} N / \mathrm{d} \log \left(M / M_{\odot}\right)$ versus mass, where $\mathrm{d} N / \operatorname{dlog}\left(M / M_{\odot}\right)$ is approximated by the number of clumps $\Delta N$ within a $\log$ arithmic mass interval $\Delta \log \left(M / M_{\odot}\right)$. Here, $\Delta \log \left(M / M_{\odot}\right)$ is constant, $\sim 0.37$. Error bars are estimated by $\sqrt{\Delta N / \Delta \log \left(M / M_{\odot}\right)}$. The continuous line represents the mass distribution fit with $\mathrm{d} N / \operatorname{dlog}\left(M / M_{\odot}\right) \propto M^{1-\alpha}$, where the spectral mass index $\alpha$ is $1.6 \pm 0.1$ for masses between $\sim 100$ and $1.3 \times 10^{3} M_{\odot}$.

Table 3. Summary of the physical properties of the identified clumps.

\begin{tabular}{ccccc}
\hline \hline & $\begin{array}{c}S_{1.2 \mathrm{~mm}} \\
\mathrm{Jy}\end{array}$ & $\begin{array}{c}\text { Diameters } \\
\mathrm{pc}\end{array}$ & $\begin{array}{c}\text { Masses } \\
M_{\odot}\end{array}$ & $\begin{array}{c}{ }^{a} \text { Densities } \\
\mathrm{cm}^{-3}\end{array}$ \\
\hline Range & $0.089-40$ & $0.2-0.6$ & $3.0-1.3 \times 10^{3}$ & $5 \times 10^{3}-4 \times 10^{5}$ \\
Total & $3.7 \times 10^{2}$ & & $1.2 \times 10^{4}$ & \\
\hline
\end{tabular}

Notes. ${ }^{(a)}$ The densities are computed assuming a mean molecular weight of $\mu=2.29$.

and range between $4 \times 10^{21}$ and $4 \times 10^{23} \mathrm{~cm}^{-2}$. Tables 3 and 5 show physical properties for each clump and a summary of them, respectively.

Figure 8 shows a plot of mass versus diameter for the clumps detected toward GMC G345.5+1.0 with reliable diameters. The dotted lines indicate constant densities at $10^{3}, 10^{4}, 10^{5}$, and $10^{6} \mathrm{~cm}^{-3}$. The majority of clumps have densities between $10^{4}$ and $10^{5} \mathrm{~cm}^{-3}$.

The physical properties of detected clumps are similar to those found in other GMCs (e.g. Mookerjea et al. 2004).

\subsection{Association with infrared emission (IRAS-MSX-Spitzer)}

Stars form in clumps, heating their surrounding dust, which reradiates at infrared wavelengths. This is illustrated in Fig. 9 that shows a strong spatial correlation between the $1.2 \mathrm{~mm}$ continuum emission and infrared emission at $21.34 \mu \mathrm{m}$ (from MSX observations). To quantify the correlation, we searched for infrared emission inside clump emission areas, using $\mathrm{MSX}^{2}$ images at $8.28,12.13,14.65$, and $21.34 \mu \mathrm{m}$ and Spitzer $^{3}$ (IRAC) images at $3.6,4.5,5.8$, and $8.0 \mu \mathrm{m}$. We find that $\sim 20 \%$ of all clumps have an infrared counterpart in all MSX and Spitzer bands (see Table 5). The rest of the clumps, $\sim 80 \%$, are not detected in all

\footnotetext{
2 http://irsa.ipac.caltech.edu/

3 http://irsa.ipac. caltech.edu/
}

MSX and Spitzer bands, particularly not in 12.13, 14.65, and $21.34 \mu \mathrm{m}$. Since $8.0 \mu \mathrm{m}$ MSX band and Spitzer IRAC bands are sensitive to the polycyclic aromatic hydrocarbon (PAH) emission and the photospheric emission from stars (e.g. Chavarría et al. 2008), clump not detected in all MSX and Spitzer bands are considered to have no counterpart at infrared wavelengths. Since both the MSX and Spitzer bands have sensitivity limits, the percentage of detections is a lower limit to the number of clumps that are forming stars, and the percentage of failed detections is an upper limit to the number of clumps that are not forming stars.

Nine clumps are associated with six IRAS point sources classified as MSFRs with luminosities $\gtrsim 10^{3} L_{\odot}$ (see Sect. 4.5 and Table 4). As Fig. 8 shows, these clumps have densities of $\sim 10^{5} \mathrm{~cm}^{-3}$, suggesting that there is a threshold density above which massive stars can form. These values are consistent with the typical density of clumps associated with MSFRs ( $\sim 10^{5} \mathrm{~cm}^{-3}$; Faúndez et al. 2004).

As Fig. 8 shows, clumps that emit detectable infrared emission tend to be more massive than remaining clumps. Clumps without infrared emission (cold or starless clumps) have a mean mass of $21 M_{\odot}$, and clumps with an infrared counterpart have a mean mass of $2.1 \times 10^{2} M_{\odot}$. Furthermore, all clumps with masses higher than $\sim 200 M_{\odot}$ have an infrared counterpart.

MSX point sources associated with clumps within the GMC G345.5+1.0 have mid-infrared colors $S_{21} / S_{8}$ from 0.9 to 30 , $S_{14} / S_{8}$ from 0.4 to 8 , and $S_{12} / S_{8}$ from 0.7 to 4 , where $S_{8}$, $S_{12}, S_{14}$, and $S_{21}$ are the flux densities at 8.28, 12.13, 14.65, and $21.34 \mu \mathrm{m}$, respectively. These ratios cover those of massive young stellar objects (MYSOs; Lumsden et al. 2002): $S_{21} / S_{8}>$ 2 and $S_{21}>S_{14}>S_{8}$. About $7 \%$ of the clumps contain MSX sources that satisfy this criterion, and these clumps have masses $\gtrsim 36 M_{\odot}$ (see Fig. 8).

The existence of clumps with and without infrared emission suggests that clumps in the GMC are at different evolutionary stages. Cold clumps have masses between 3.0 and $1.9 \times 10^{2} M_{\odot}$, where the most massive ones are possible progenitors of MSFRs. For example, we estimate that the least massive clump associated with a MYSO has a mass of $\sim 36 M_{\odot}$, and we identify seven cold clumps with densities $\gtrsim 10^{5} \mathrm{~cm}^{-3}$ and masses $\gtrsim 36 M_{\odot}$, which will eventually collapse to form high-mass stars.

Do clumps form single stars? One way to assess this is to compare the slope of the clump mass distribution with that of the stellar initial mass function (IMF) (e.g. Motte et al. 1998; Lada et al. 2007). Equal slopes would indicate that the origin of the stellar IMF has its direct roots in the origin of the clump mass distribution. The spectral mass index $\alpha$ of the clump mass distribution determined here is consistent with that of other investigations (e.g. Muñoz et al. 2007), but differs from that estimated for the stellar IMF of the solar neighborhood for stellar masses higher than $0.5 M_{\odot}(\alpha \sim 2.35$; e.g. Kroupa 2002). This suggests that the detected clumps do not directly form stars, and other processes are necessary to determine the stellar initial masses, such as the fragmentation of clumps, mainly of the most massive ones. Figure 6 compares the IMF spectral mass index with the clump mass distribution.

\subsection{Dust properties of massive-star forming regions associated with clumps and IRAS point sources}

Regions of massive star formation are embedded in massive clumps, and the intense Lyman flux produced by them heats the surrounding dust, which re-emits mainly at far infrared wavelengths with characteristic colors. To study the physical 


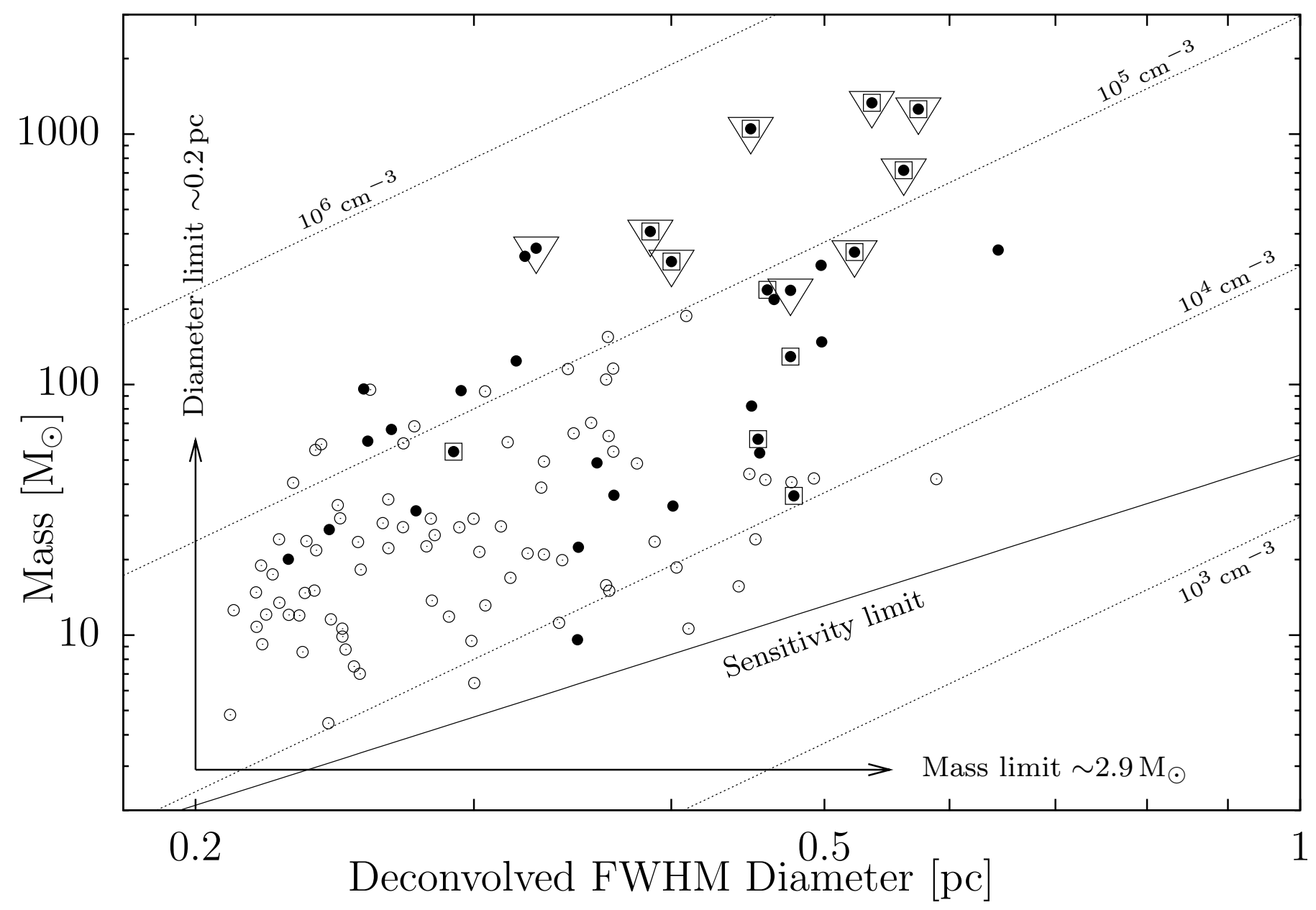

Fig. 8. Mass versus diameter for the clumps detected toward the GMC G345.5+1.0 in $1.2 \mathrm{~mm}$ continuum emission with reliable diameters. Filled circles indicate clumps detected in infrared MSX and Spitzer bands. Open circles indicate clumps that do not have an infrared counterpart. Triangles indicate clumps associated with MSFR-IRAS sources, which have luminosities $>10^{3} L_{\odot}$. Boxes indicate clumps associated with MSX sources that satisfy MYSO candidate criterion (Lumsden et al. 2002). Arrows mark detection limits for masses $\left(\sim 2.9 M_{\odot}\right)$ and diameters $(\sim 0.2 \mathrm{pc})$. The continuous line indicates the detectable mass as a function of diameter (sensitivity limit), considering an intensity limit of five rms $\left(\sim 0.1 \mathrm{Jy} \mathrm{beam}^{-1}\right)$. Dotted lines indicate mean densities at $10^{3}, 10^{4}, 10^{5}$ and $10^{6} \mathrm{~cm}^{-3}$. The densities are computed assuming a mean molecular weight of $\mu=2.29$.

Table 4. SED models for MSFRs associated with IRAS point sources and massive clumps detected in 1.2 mm continuum emission.

\begin{tabular}{lccccccccc}
\hline \hline IRAS & Clumps & \multicolumn{3}{c}{ Cold } & \multicolumn{3}{c}{ Warm } & Cold mass & Luminosity \\
& & $\begin{array}{c}T_{\text {dust }} \\
\mathrm{K}\end{array}$ & $\begin{array}{c}D_{\text {dust }} \\
\mathrm{pc}\end{array}$ & $\begin{array}{c}N_{\text {gas }} \\
10^{23} \mathrm{~cm}^{-2}\end{array}$ & $\begin{array}{c}T_{\text {dust }} \\
\mathrm{K}\end{array}$ & $\begin{array}{c}D_{\text {dust }} \\
\mathrm{pc}\end{array}$ & $\begin{array}{c}N_{\text {gas }} \\
10^{22} \mathrm{~cm}^{-2}\end{array}$ & $10^{3} M_{\odot}$ & $10^{4} L_{\odot}$ \\
\hline $16562-3959$ & 1 & 32 & 0.67 & 1.6 & 194 & 0.03 & 0.69 & 1.0 & 5.3 \\
$17009-4042$ & 2 & 29 & 0.86 & 1.6 & 202 & 0.03 & 0.51 & 1.7 & 4.7 \\
$16533-4009$ & $3,7,10$ & 25 & 0.79 & 3.2 & 205 & 0.07 & 2.7 & 2.8 & 9.4 \\
$17008-4040$ & 4 & 28 & 0.68 & 1.5 & 205 & 0.03 & 0.57 & 1.0 & 2.9 \\
$16571-4029$ & 5,9 & 34 & 0.58 & 1.3 & 209 & 0.03 & 0.65 & 0.64 & 5.3 \\
$16596-4012$ & 12 & 22 & 0.34 & 2.2 & 203 & 0.008 & 0.49 & 0.36 & 0.22 \\
\hline
\end{tabular}

Notes. Each model consists of two dust components with equal densities at different temperatures (cold and warm components). Column 1 shows names of IRAS point sources; Col. 2, names of clumps; in Cols. 3 to 8, we show the fitted physical parameters: dust temperature, diameter, and column density, respectively, for the cold (Cols. 3-5) and warm (Cols. 6-8) components; Col. 9, masses of the cold component; and Col. 10, total luminosities. 


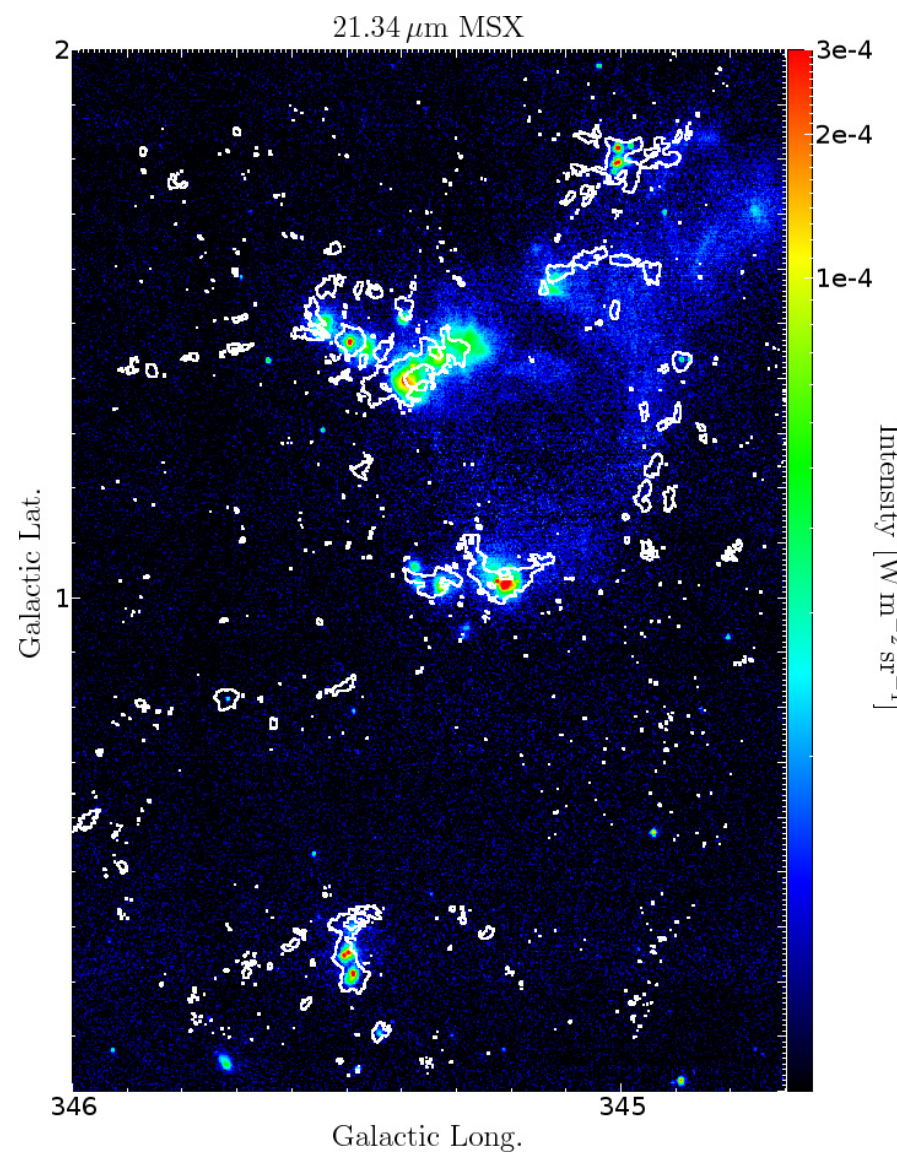

Fig. 9. Image in $21.34 \mu \mathrm{m}$ from MSX observations toward GMC G345.5+1.0 with contours of $1.2 \mathrm{~mm}$ continuum emission at three times $\mathrm{rms}, \sim 0.06 \mathrm{Jy} \mathrm{beam}^{-1}$.

properties of dust in these regions, we examine the spectral energy distributions (SEDs) of MSFRs associated with IRAS point sources and clumps detected here, assuming that their emissions are from dust.

Within the GMC, there are eight MSFRs associated with IRAS-CS sources (Table 2). We added one more source, IRAS 16533-4009, which is embedded in $1.2 \mathrm{~mm}$ continuum emission, has a high luminosity, $\sim 9 \times 10^{4} L_{\odot}$, and increasing IRAS flux densities from 12 to $100 \mu \mathrm{m}$; however it does not satisfy the far-infrared color criterion defined by Wood \& Churchwell (1989), since its flux density in $25 \mu \mathrm{m}$ is an upper limit. Figure 11 shows $8.0 \mu \mathrm{m}$ images from Spitzer data with contours of $1.2 \mathrm{~mm}$ continuum emission for all these sources. For six of these objects, the Spitzer emission is embedded within $1.2 \mathrm{~mm}$ continuum emission. For more reliable estimates in our SED study, we only consider these six sources: IRAS 16533 4009, IRAS 16562-3959, IRAS 16571-4029, IRAS 16596-4012, IRAS 17008-4040, and IRAS 17009-4042.

Figure 12 displays the six SEDs constructed using our observations in $1.2 \mathrm{~mm}$ continuum emission, infrared data at 12 , 25,60 , and $100 \mu \mathrm{m}$ from the IRAS Point Source Catalog (version 2.0), and at 8.3, 12.1, 14.7 and $21.3 \mu \mathrm{m}$ from the MSX Point Source Catalog (version 2.3). Because observations were performed using different beam sizes, for IRAS $\sim 300^{\prime \prime}$, for MSX

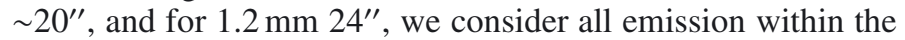
IRAS beam; thus, two SEDs are associated with more than one $1.2 \mathrm{~mm}$ clump.

The SEDs of MSFRs can be modeled as several dust components at different temperatures (e.g. Faúndez et al. 2004; Morales et al. 2009). Because of the shape of the six SEDs, we model

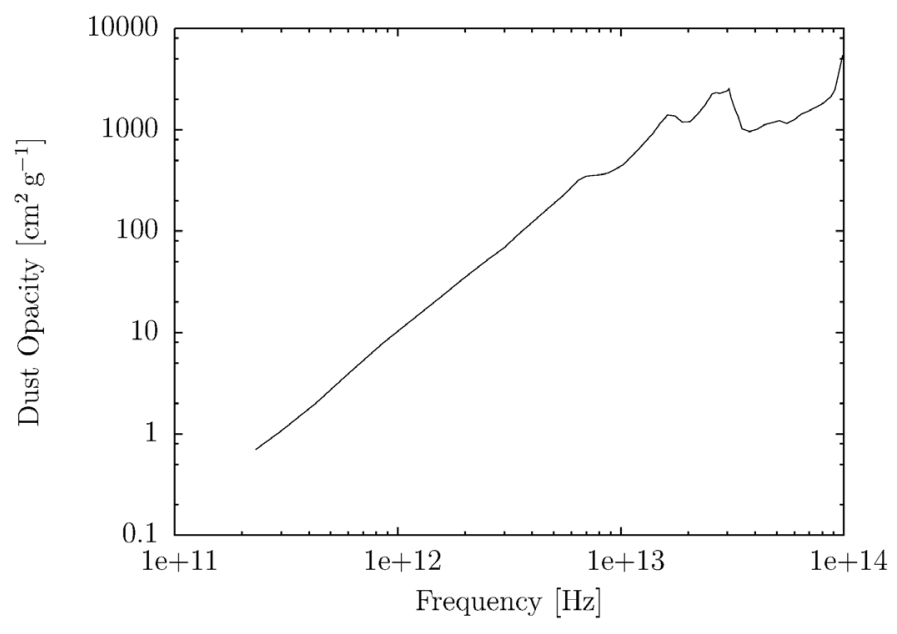

Fig. 10. Dust opacity spectrum utilized in the SED models. It was estimated by Ossenkopf \& Henning (1994) ${ }^{a}$, assuming a Mathis-RumplNordsieck initial size distribution with thin ice mantles and $10^{5} \mathrm{yr}$ of coagulation at a gas density of $10^{5} \mathrm{~cm}^{-3} \cdot{ }^{a}$ http: //vizier.u-strasbg . fr/viz-bin/VizieR, J/A+A/291/943/table1.

them as two dust components at different temperatures, cold and warm components, including the absorption of the radiation by assuming that the warm component is embedded in the cold one. The total flux density, $S_{v}^{\text {total }}$, at frequency $v$ is approximated by

$S_{v}^{\text {total }} \sim S_{v}^{\text {cold }}+S_{v}^{\text {warm }}$,

where

$S_{v}^{\text {cold }}=\Omega^{\text {cold }} B_{v}\left(T_{\text {dust }}^{\text {cold }}\right)\left(1-\exp \left(-\tau_{v}^{\text {cold }}\right)\right)$

and

$S_{v}^{\text {warm }}=\Omega^{\text {warm }} B_{v}\left(T_{\text {dust }}^{\text {warm }}\right)\left(1-\exp \left(-\tau_{v}^{\text {warm }}\right)\right) \exp \left(-\tau_{v}^{\text {cold }} / 2\right)$.

The parameters $S_{v}^{\text {cold }}, \Omega^{\text {cold }}, T_{\text {dust }}^{\text {cold }}$, and $\tau_{v}^{\text {cold }}$ are the flux density, the solid angle, the dust temperature, and the optical depth of the cold component, respectively, and $S_{v}^{\text {warm }}, \Omega^{\text {warm }}, T_{\text {dust }}^{\text {warm }}$, and $\tau_{v}^{\text {warm }}$ are the flux density, the solid angle, the dust temperature, and the optical depth of the warm component, respectively. In addition, $B_{v}\left(T_{\text {dust }}^{\text {cold }}\right)$ and $B_{v}\left(T_{\text {dust }}^{\text {warm }}\right)$ are the Planck function at dust temperatures $T_{\text {dust }}^{\text {cold }}$ and $T_{\text {dust }}^{\text {warm }}$, respectively. For both components, $\Omega$ can be expressed as

$\Omega=\pi(\theta / 2)^{2}$,

where $\theta$ is the angular diameter. The optical depths are given by (e.g. Evans 1999)

$\tau_{v}^{\text {cold }}=N_{\text {dust }}^{\text {cold }} k_{v}$ and $\quad \tau_{v}^{\text {warm }}=N_{\text {dust }}^{\text {warm }} k_{v}$,

where $N_{\text {dust }}^{\text {cold }}$ and $N_{\text {dust }}^{\text {warm }}$ are the dust column densities in $\mathrm{g} \mathrm{cm}^{-2}$ for the cold and warm components, and $k_{v}$ is the dust opacity. We use dust opacities estimated by Ossenkopf \& Henning (1994) for protostellar cores. They computed opacities considering the Mathis-Rumpl-Nordsieck (MRN) distribution for the diffuse interstellar medium (Draine \& Lee 1984) as the initial size distribution for dust, without and with ice (thin and thick), and without and with coagulation (after $10^{5}$ years for densities between $10^{5}$ $10^{8} \mathrm{~cm}^{-3}$ ). In the case of regions with ice depletion produced by the heating of central sources, they recommended opacities for the model with thin ice mantles and coagulation for a density of $10^{5} \mathrm{~cm}^{-3}$. These dust opacities are shown in Fig. 10, for frequencies between $\sim 2.3 \times 10^{11}$ and $10^{14} \mathrm{~Hz}$. 


\section{Embedded sources}
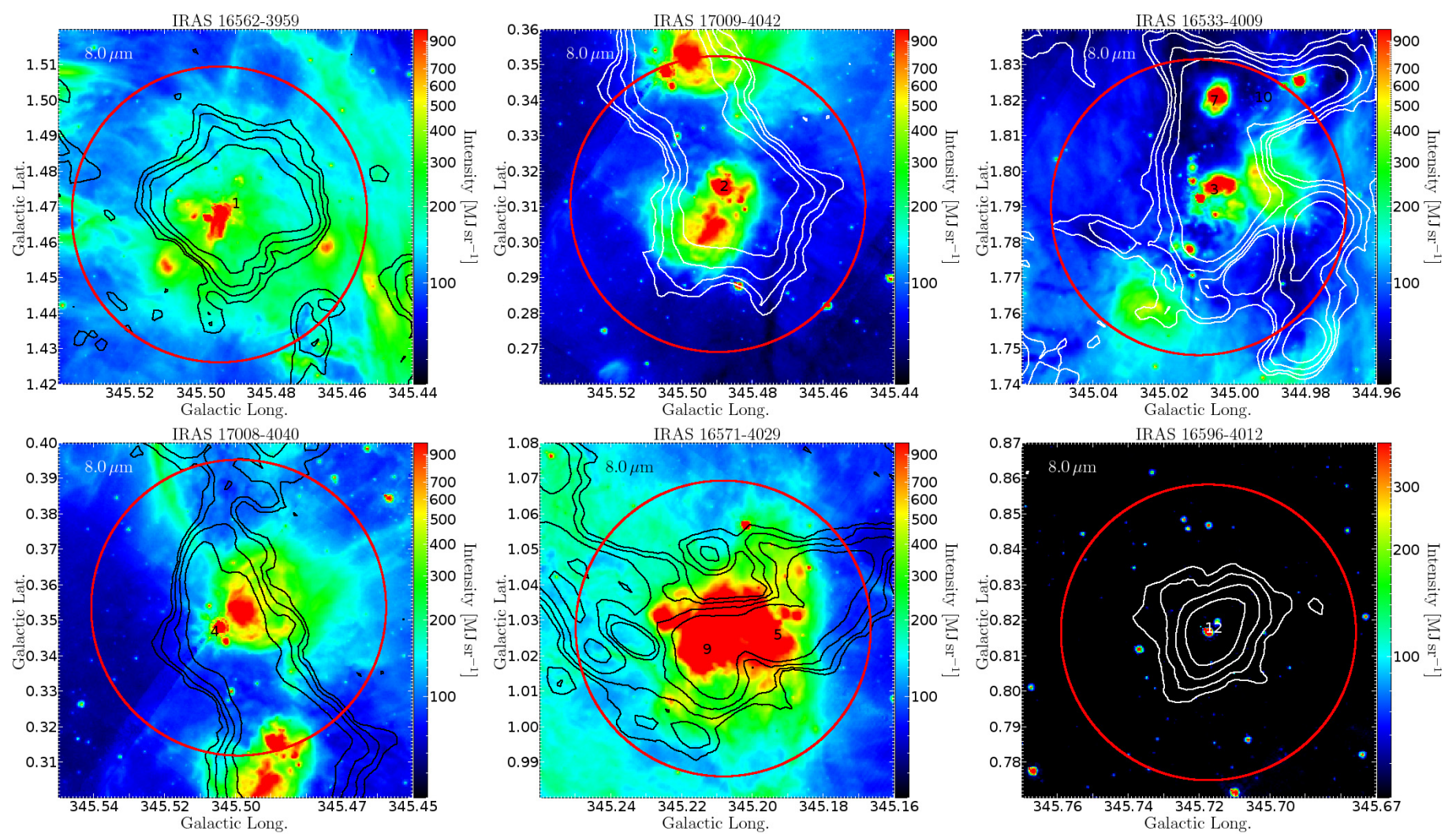

\section{Partially embedded sources}
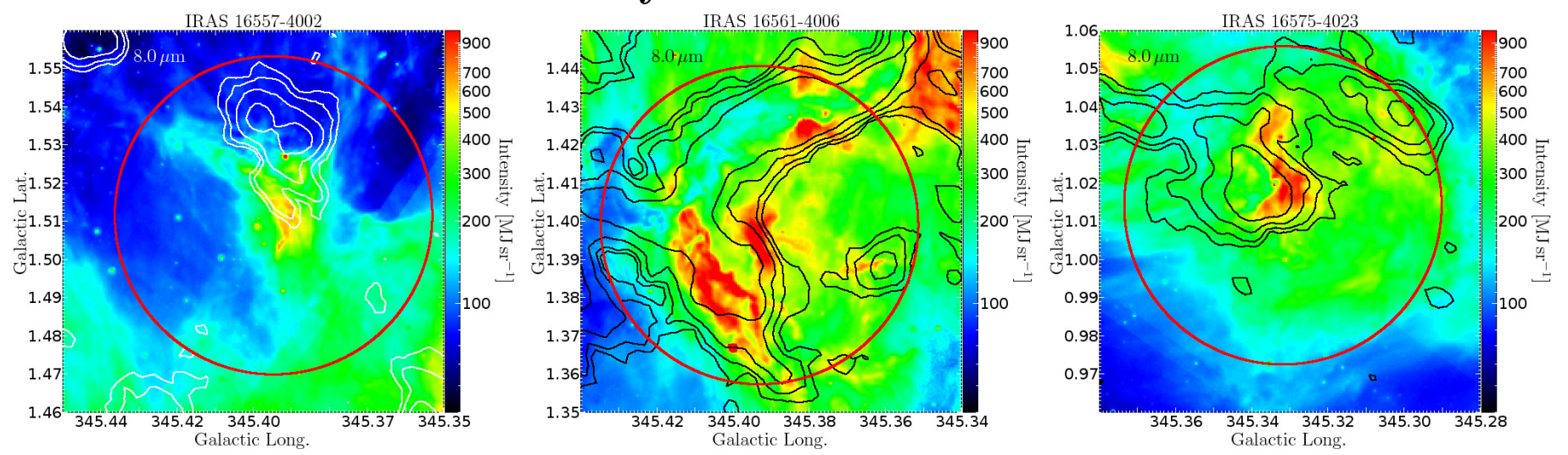

Fig. 11. Images of $8.0 \mu \mathrm{m}$ emission (Spitzer data) toward clumps detected in $1.2 \mathrm{~mm}$ continuum emission and associated with IRAS point sources. Contours represent $1.2 \mathrm{~mm}$ continuum emission at $0.06,0.12,0.24$, and $0.48 \mathrm{Jy} \mathrm{beam}^{-1}\left(\mathrm{rms}\right.$ is $\left.0.02 \mathrm{Jy} \mathrm{beam}^{-1}\right)$. IRAS source names are given at the top of each image, and clump numbers are indicated at the peak of $1.2 \mathrm{~mm}$ continuum emission. Red circles are centered on the coordinates of IRAS point sources, with diameters of 5' (an approximation of the angular resolution of IRAS observations at $100 \mu \mathrm{m}$ ).

Thus, in our SED model, each dust component has three values to fit of $T_{\text {dust }}, \theta$, and $N_{\text {dust }}$. However, the dust column density of the warm component, $N_{\text {dust }}^{\text {warm }}$, is difficult to estimate, because it is more sensitive to the emission in the Rayleigh-Jeans part of the spectrum $\left(h v \ll k T_{\text {warm }}\right)$, where the emission is dominated by the cold component. To overcome this problem, we assume that the two components have equal densities, thus

$N_{\text {dust }}^{\text {warm }} \sim \frac{\theta_{\text {dust }}^{\text {warm }}}{\theta_{\text {dust }}^{\text {cold }}} N_{\text {dust }}^{\text {cold }}$.

Given the simplicity of the SED model and the poor sensitivity of the data to $N_{\text {dust }}^{\text {warm }}$, a more realistic density distribution is unnecessary.
To enable a more reliable comparison, angular diameters are converted into spatial diameters, and dust column densities to gas column densities. Thus

$D_{\text {dust }}^{\text {cold }}=\mathrm{d} \theta_{\text {dust }}^{\text {cold }}$,

$D_{\text {dust }}^{\text {hot }}=\mathrm{d} \theta_{\text {dust }}^{\text {warm }}$,

and

$N_{\mathrm{gas}}^{\text {cold }}=\frac{N_{\mathrm{dust}}^{\text {cold }} R_{\mathrm{gd}}}{\mu m_{\mathrm{H}}}$,

where $\theta_{\text {dust }}^{\text {cold }}$ and $\theta_{\text {dust }}^{\text {warm }}$ are in radians. In this way, our model has five variables: dust temperatures and sizes for the two components, and gas column density for the cold component. 
Clump 1

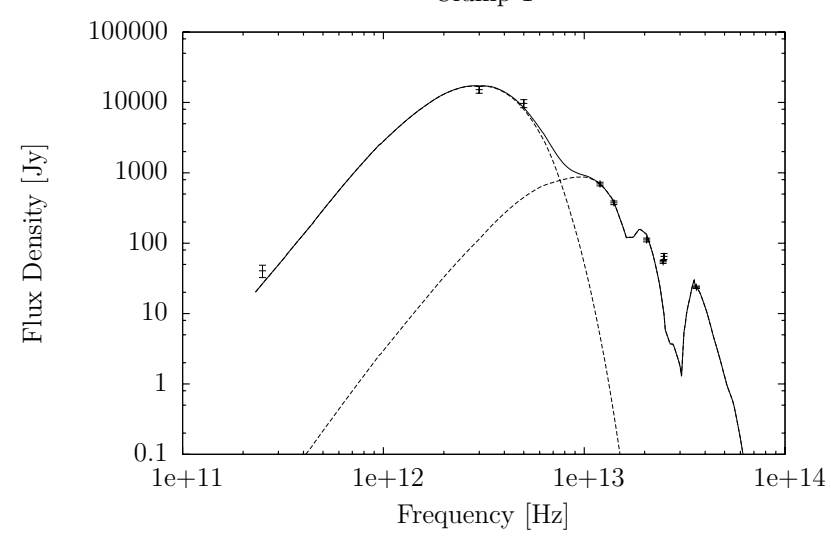

Clumps $3,7 \& 10$

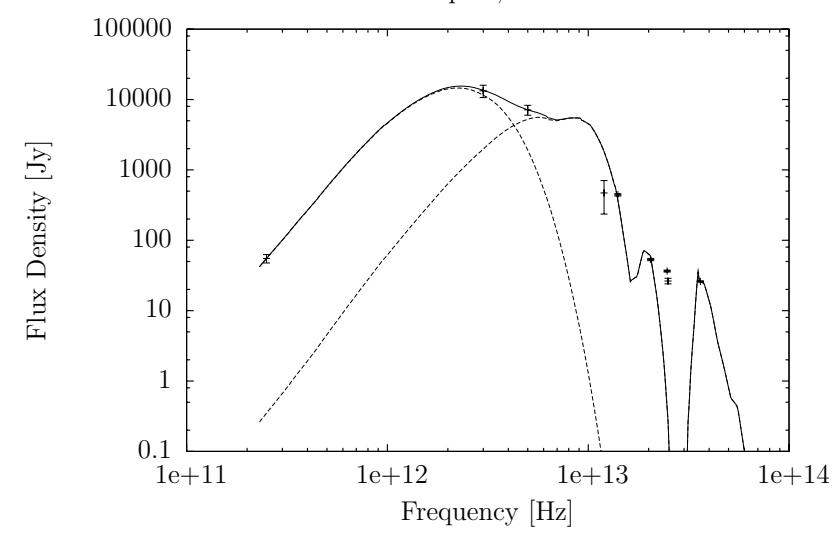

Clumps $5 \& 9$

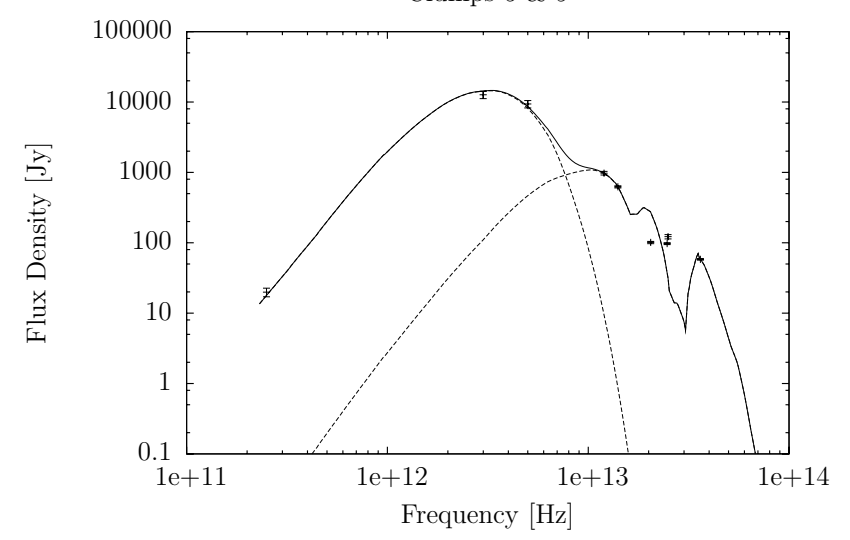

Clump 2

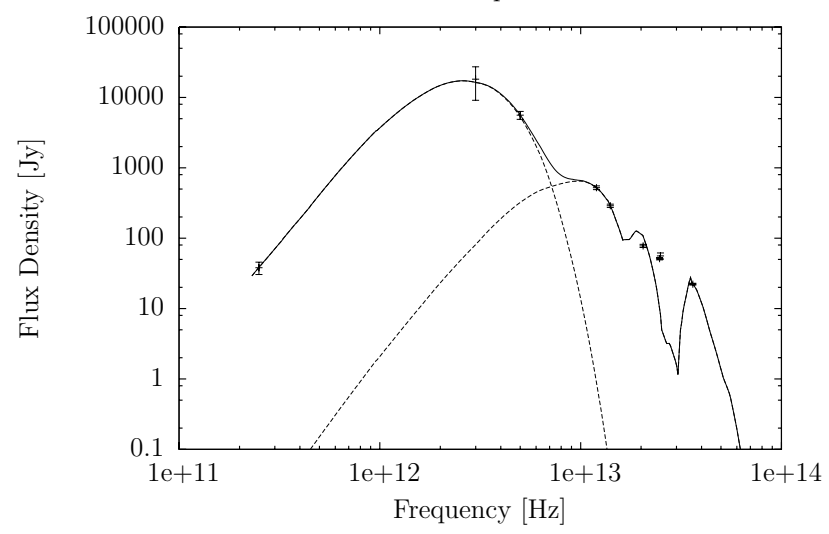

Clump 4

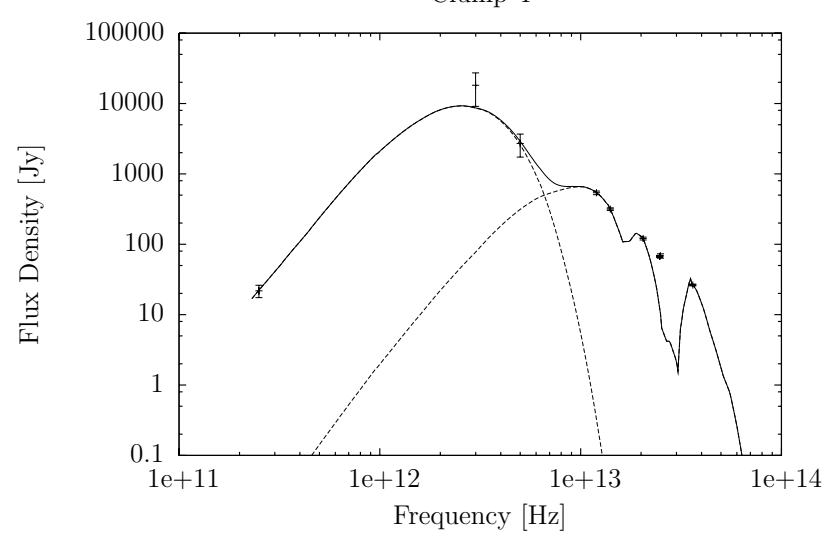

Clump 12

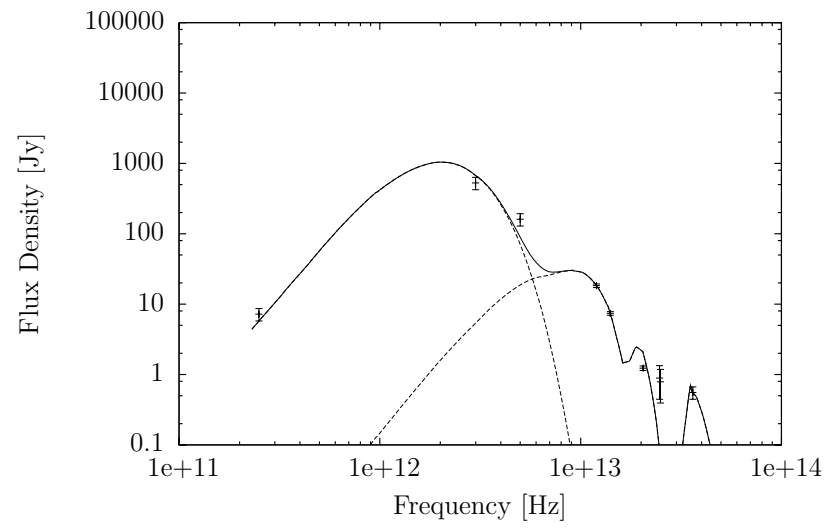

Fig. 12. The SEDs of massive-star forming regions associated with massive clumps detected in $1.2 \mathrm{~mm}$ continuum emission; top labels show names of the clumps. Dots with error bars are flux densities estimated from SIMBA, IRAS, and MSX observations. Each SED is modeled with two dust components at different temperatures (physical parameters for each model are in Table 4); drawn lines are the total flux density of the two dust components, and dashed lines are the contributions of each dust component.

Table 4 and Fig. 12 display the results of the fits. The mean dust temperatures of each component are $28 \pm 5 \mathrm{~K}$ (cold) and $200 \pm 10 \mathrm{~K}$ (warm). The sizes and column densities of the cold component agree with those estimated by $1.2 \mathrm{~mm}$ continuum: sizes vary by a factor of $0.7-1.5$ and column densities vary by a factor of $0.5-3$. Estimates of luminosities, from the integration of SED models, are $>10^{3} L_{\odot}$. Given the sizes and column densities, the total mass is dominated by the cold component ( $\sim 99 \%$ of the total mass), and is similar to that estimated from the $1.2 \mathrm{~mm}$ continuum emission, varying by a factor of $0.8-1.6$.
Dust characteristics of clumps associated with MSFRs estimated in this paper are consistent with previous works (e.g. Faúndez et al. 2004; Molinari et al. 2000; Molinari et al. 2008), where the cold dust temperature in regions of massive star formation is $\sim 30 \mathrm{~K}$.

The SED models have a discrepancy with data in $\sim 12 \mu \mathrm{m}$ bands (see Fig. 12), which can be produced by not considering the PAH emission in the SED models (e.g. van Dishoeck 2004), or by an excess in the dust opacities utilized at these wavelengths, affecting the modeled radiation from the hot component that is absorbed by the cold one. 


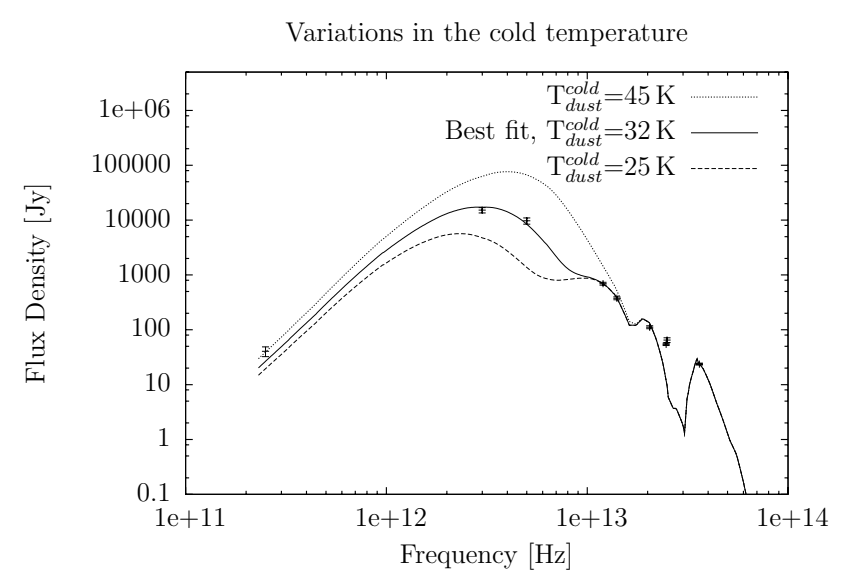

Variations in the warm temperature

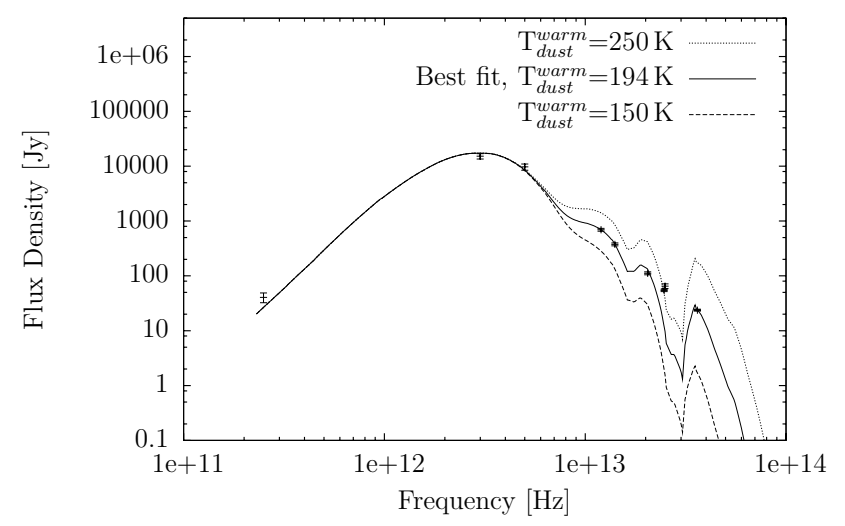

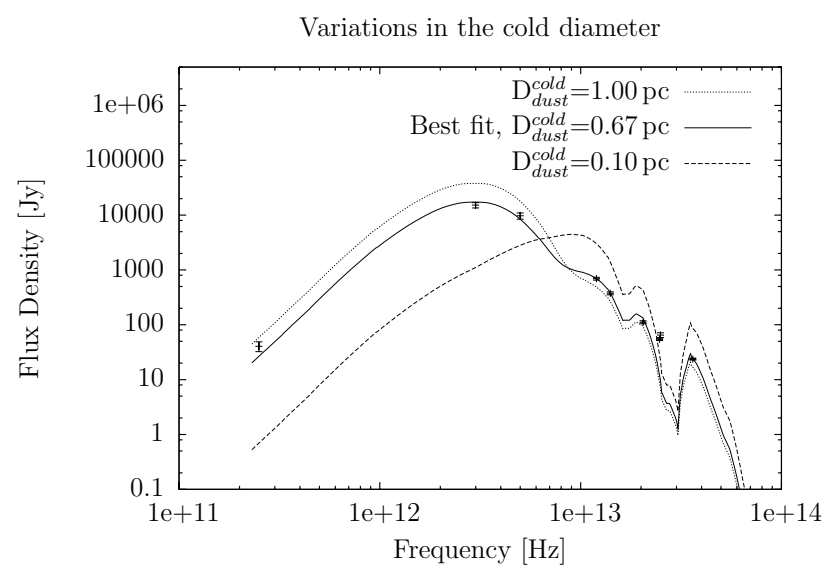

Variations in the warm diameter

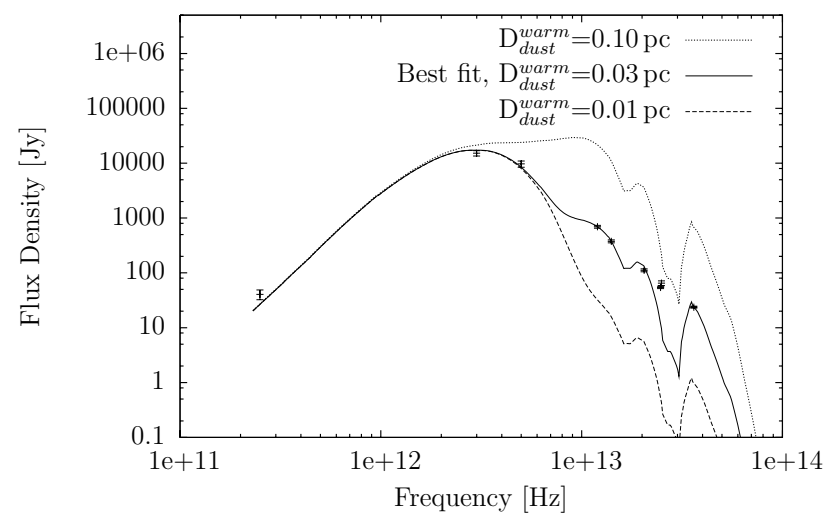

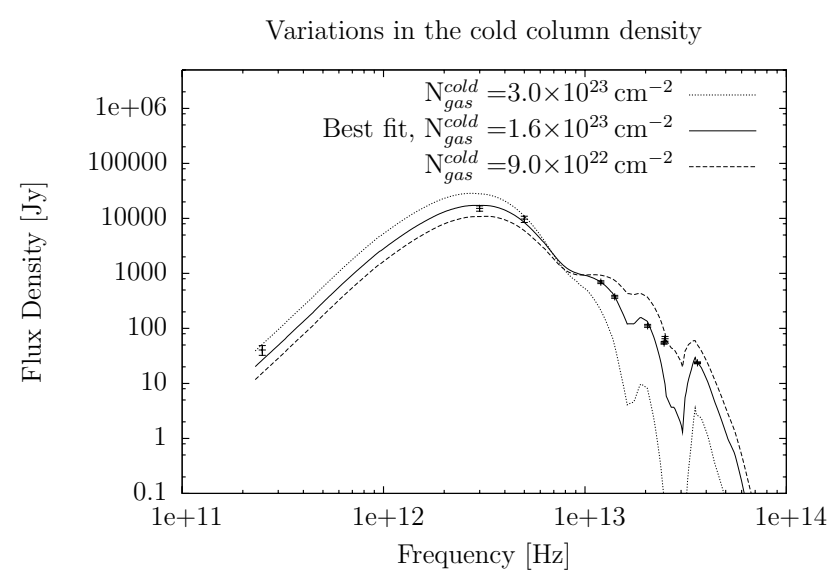

Fig. 13. Dependence of the SED model on variations in the fitted parameters. Plots show the SED for clump 1 with the best-fit model (see Table 4 ) and variations in each parameter, for $T_{\text {dust }}^{\text {cold }}, D_{\text {dust }}^{\text {cold }}, T_{\text {dust }}^{\text {warm }}, D_{\text {dust }}^{\text {warm }}$ and $N_{\text {gas }}^{\text {cold }}$, when increasing and decreasing the best-fit value.

The dependence of SED models on variations in the fitted parameters is shown in Fig. 13, which displays the SED for clump 1, or IRAS 16562-3959, with the best-fit model and models with variations in the fitted parameters $T_{\text {dust }}^{\text {cold }}, D_{\text {dust }}^{\text {cold }}, T_{\text {dust }}^{\text {warm }}$, $D_{\text {dust }}^{\text {warm }}$, and $N_{\text {gas }}^{\text {cold }}$. For each dust component, variations in its temperature affect both its peak emission frequency and its luminosity, both values increasing as the temperature increases. Variations in either $D_{\text {dust }}^{\text {cold }}$ or $N_{\text {gas }}^{\text {cold }}$ affect the luminosities of the two components; when $D_{\text {dust }}^{\text {cold }}$ or $N_{\text {gas }}^{\text {cold }}$ increases, the luminosity of the cold dust component increases, whereas radiation absorption of the warm component also increases, reducing its luminosity.

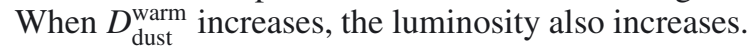

\section{Conclusions}

We have robustly detected the whole of the GMC G345.5+1.0 in $1.2 \mathrm{~mm}$ continuum emission at a spatial resolution of $0.2 \mathrm{pc}$, and conclude that:

- The GMC is fragmented. We have identified 201 clumps, which have beam-corrected diameters between 0.2 and $0.6 \mathrm{pc}$, masses between 3.0 and $1.3 \times 10^{3} M_{\odot}$, and densities between $5 \times 10^{3}$ and $4 \times 10^{5} \mathrm{~cm}^{-3}$.

- The total mass of the clumps is $\sim 1.2 \times 10^{4} M_{\odot}$, and after comparing with the total mass of the GMC of $\sim 6.5 \times 10^{5} M_{\odot}$, we inferred that the efficiency in forming these clumps is $\sim 0.02$. 
- The clump mass distribution is well-fitted by a power law $\mathrm{d} N / \mathrm{d} M \propto M^{-\alpha}$, where the spectral mass index $\alpha$ is $1.7 \pm$ 0.1 . The total mass is dominated by massive clumps, but the population is dominated by clumps with low masses.

- The spectral mass index of the clump mass distribution is different from that of the stellar IMF. Thus our detected clumps are probably not the direct progenitors of single stars.

- Comparing with MSX and Spitzer (IRAC-bands) observations, $20 \%$ of the clumps have an infrared counterpart in all MSX and Spitzer bands. The remaining clumps, $\sim 80 \%$, are considered to have no counterpart at infrared wavelengths. The percentage of detection is a lower limit to the number of clumps forming stars, while the percentage of no detections is an upper limit to the number of clumps that are not forming stars.

- Regions of massive-star formation within the cloud, associated with IRAS point sources, have SEDs that can be modeled with two dust components at different mean temperatures of $28 \pm 5$ and $200 \pm 10 \mathrm{~K}$.

Acknowledgements. C.L. acknowledges partial support from the GEMINICONICYT FUND, project number 32070020, and ESO-University of Chile Student Fellowship. This work was supported by the Chilean Center for Astrophysics FONDAP No. 15010003 and by Center of Excellence in Astrophysics and Associated Technologies PFB 06.

\section{References}

Alvarez, H., May, J., \& Bronfman, L. 1990, ApJ, 348, 495

Bains, I., Wong, T., Cunningham, M., et al. 2006, MNRAS, 367, 1609 Ballesteros-Paredes, J., Gazol, A., Kim, J., et al. 2006, ApJ, 637, 384

Ballesteros-Paredes, J., Klessen, R. S., Mac Low, M.-M., \& Vázquez-Semadeni, E. 2007, Protostars and Planets V, ed. B. Reipurth, D. Jewitt, \& K. Keil (University of Arizona Press), 63

Beltrán, M. T., Brand, J., Cesaroni, R., et al. 2006, A\&A, 447, 221

Bohlin, R. C., Savage, B. D., \& Drake, J. F. 1978, ApJ, 224, 132
Bonnell, I. A., Larson, R. B., \& Zinnecker, H. 2007, Protostars and Planets V, ed. B. Reipurth, D. Jewitt, \& K. Keil (University of Arizona Press), 149

Bronfman, L., Alvarez, H., Cohen, R. S., \& Thaddeus, P. 1989, ApJ, 71, 481

Bronfman, L., Nyman, L.-А., \& May, J. 1996, A\&A, 115, 81

Bronfman, L., Casassus, S., May, J., \& Nyman, L.-A. 2000, A\&A, 358, 521

Chavarría, L. A., Allen, L. E., Hora, J. L., Brunt, C. M., \& Fazio, G. G. 2008, ApJ, 682, 445

Dame, T. M., Elmegreen, B. G., Cohen, R. S., \& Thaddeus, P. 1986, ApJ, 305, 892

Draine, B. T., \& Lee, H. M. 1984, ApJ, 285, 89

Evans, N. J. 1999, ARA\&A, 37, 311

Faúndez, S., Bronfman, L., Garay, G., et al. 2004, A\&A, 426, 97

Garay, G., Faúndez, S., Mardones, D., et al. 2004, ApJ, 610, 313

Grabelsky, D. A., Cohen, R. S., Bronfman, L., \& Thaddeus, P. 1988, ApJ, 331, 181

Hildebrand, R. H. 1983, QJRAS, 24, 267

Hill, T., Burton, M. G., Minier, V., et al. 2005, MNRAS, 363, 405

Hunter, S. D., Bertsch, D. L., Catelli, J. R., et al. 1997, ApJ, 481, 205

Klessen, R. S., Burkert, A., \& Bate, M. R. 1998, ApJ, 501, 205

Kroupa, P. 2002, Science, 295, 82

Lada, C. J., Alves, J. F., \& Lombardi, M. 2007, Protostars and Planets V, ed. B. Reipurth, D. Jewitt, \& K. Keil (University of Arizona Press), 3

Li, Y., Mac Low, M.-M., \& Klessen, R.S. 2005, ApJ, 626, 823

Lumsden, S. L., Hoare, M. G., Oudmaijer, R. D., \& Richards, D. 2002, MNRAS, 336,621

Molinari, S., Brand, J., Cesaroni, R., \& Palla, F. 2000, A\&A, 355, 617

Molinari, S., Pezzuto, S., Cesaroni, R., et al. 2008, A\&A, 481, 345

Mookerjea, B., Kramer, C., Nielbock, M., \& Nyman, L.-Å. 2004, A\&A, 426, 119

Morales, E. F. E., Mardones, D., Garay, G., et al. 2009, ApJ, 698, 488

Motte, F., André, P., \& Neri, R. 1998, A\&A, 336, 150

Motte, F., Bontemps, S., Schilke, P., et al. 2007, A\&A, 476, 1243

Muñoz, D. J., Mardones, D., Garay, G., \& Rebolledo, D. 2007, ApJ, 668, 906

Ossenkopf, V., \& Henning, Th. 1994, A\&A, 291, 943

Panagia, N. 1973, ApJ, 78, 929

Sanders, D. B., Scoville, N. Z., \& Solomon, P. M. 1985, ApJ, 289, 373

Solomon, P. M., Rivolo, A. R., Barrett, J., \& Yahil, A. 1987, ApJ, 319, 730

Williams, J. P., \& McKee, C. F. 1997, ApJ, 476, 166

Williams, J. P., De Geus, E. J., \& Blitz, L. 1994, ApJ, 428, 693

Williams, J. P., Blitz, L., \& McKee, C. F. 2000, Protostars and Planets IV, ed. V. Mannings, A. P. Boss, \& S. S. Russell (University of Arizona Press), 97 van Dishoeck 2004, ARA\&A, 42, 119

Wood, D. O. S., \& Churchwell, E. 1989, ApJ, 340, 265

Zinnecker, H., \& York, H. 2007, ARA\&A, 45, 481 
Table 5. Properties of identified clumps in the GMC G345.5+1.0.

\begin{tabular}{|c|c|c|c|c|c|c|c|c|}
\hline Name & $\begin{array}{l}\text { Galactic } \\
\text { long. }\end{array}$ & $\begin{array}{l}\text { coord. } \\
\text { lat. }\end{array}$ & $\begin{array}{c}S_{1.2 \mathrm{~mm}} \\
\text { Jy }\end{array}$ & $\begin{array}{c}\text { Diameter } \\
\text { pc }\end{array}$ & $\begin{array}{c}\text { Mass } \\
M_{\odot}\end{array}$ & $\begin{array}{c}\text { Density } \\
\mathrm{cm}^{-3}\end{array}$ & $\begin{array}{c}\text { Column density } \\
\mathrm{cm}^{-2}\end{array}$ & $\begin{array}{c}\text { Infrared } \\
\text { counterpart }\end{array}$ \\
\hline 1 & 345.490 & 1.471 & 40 & 0.5 & $1.3 \times 10^{3}$ & $2.9 \times 10^{5}$ & $3.2 \times 10^{23}$ & $\mathrm{Y}$ \\
\hline 2 & 345.488 & 0.316 & 38 & 0.6 & $1.3 \times 10^{3}$ & $2.3 \times 10^{5}$ & $2.7 \times 10^{23}$ & Y \\
\hline 3 & 345.006 & 1.795 & 32 & 0.5 & $1.1 \times 10^{3}$ & $3.9 \times 10^{5}$ & $3.6 \times 10^{23}$ & $\mathrm{Y}$ \\
\hline 4 & 345.506 & 0.347 & 22 & 0.6 & $7.2 \times 10^{2}$ & $1.4 \times 10^{5}$ & $1.6 \times 10^{23}$ & $\mathrm{Y}$ \\
\hline 5 & 345.193 & 1.026 & 11 & 0.3 & $3.5 \times 10^{2}$ & $3.3 \times 10^{5}$ & $2.3 \times 10^{23}$ & $\mathrm{Y}$ \\
\hline 6 & 345.385 & 1.428 & 9.8 & 0.3 & $3.3 \times 10^{2}$ & $3.3 \times 10^{5}$ & $2.2 \times 10^{23}$ & $\mathrm{Y}$ \\
\hline 7 & 345.006 & 1.820 & 12 & 0.4 & $4.1 \times 10^{2}$ & $2.4 \times 10^{5}$ & $1.9 \times 10^{23}$ & $\mathrm{Y}$ \\
\hline 8 & 345.179 & 1.043 & 9.1 & 0.5 & $3.0 \times 10^{2}$ & $8.2 \times 10^{4}$ & $8.4 \times 10^{22}$ & $\mathrm{Y}$ \\
\hline 9 & 345.213 & 1.022 & 9.4 & 0.4 & $3.1 \times 10^{2}$ & $1.6 \times 10^{5}$ & $1.3 \times 10^{23}$ & Y \\
\hline 10 & 344.992 & 1.821 & 10 & 0.5 & $3.4 \times 10^{2}$ & $8.0 \times 10^{4}$ & $8.6 \times 10^{22}$ & Y \\
\hline 11 & 345.115 & 1.592 & 6.6 & 0.5 & $2.2 \times 10^{2}$ & $7.4 \times 10^{4}$ & $7.0 \times 10^{22}$ & Y \\
\hline 12 & 345.716 & 0.818 & 7.2 & 0.5 & $2.4 \times 10^{2}$ & $7.5 \times 10^{4}$ & $7.3 \times 10^{22}$ & Y \\
\hline 13 & 345.337 & 1.018 & 7.2 & 0.5 & $2.4 \times 10^{2}$ & $8.3 \times 10^{4}$ & $7.8 \times 10^{22}$ & Y \\
\hline 14 & 344.976 & 1.783 & 5.7 & 0.4 & $1.9 \times 10^{2}$ & $9.3 \times 10^{4}$ & $7.8 \times 10^{22}$ & $\mathrm{~N}$ \\
\hline 15 & 344.980 & 1.752 & 3.5 & 0.3 & $1.2 \times 10^{2}$ & $9.6 \times 10^{4}$ & $6.8 \times 10^{22}$ & $\mathrm{~N}$ \\
\hline 16 & 345.367 & 1.434 & 3.8 & 0.3 & $1.2 \times 10^{2}$ & $1.3 \times 10^{5}$ & $8.5 \times 10^{22}$ & Y \\
\hline 17 & 345.296 & 1.452 & 10 & 0.6 & $3.4 \times 10^{2}$ & $4.4 \times 10^{4}$ & $5.8 \times 10^{22}$ & Y \\
\hline 18 & 345.401 & 1.419 & 4.7 & 0.4 & $1.6 \times 10^{2}$ & $1.1 \times 10^{5}$ & $8.1 \times 10^{22}$ & $\mathrm{~N}$ \\
\hline 19 & 345.418 & 1.401 & 2.9 & 0.3 & 95 & $1.9 \times 10^{5}$ & $1.0 \times 10^{23}$ & $\mathrm{~N}$ \\
\hline 20 & 345.395 & 1.380 & 4.5 & 0.5 & $1.5 \times 10^{2}$ & $4.0 \times 10^{4}$ & $4.1 \times 10^{22}$ & Y \\
\hline 21 & 345.411 & 1.406 & 2.9 & 0.3 & 96 & $1.9 \times 10^{5}$ & $1.0 \times 10^{23}$ & Y \\
\hline 22 & 345.434 & 1.405 & 2.8 & 0.3 & 94 & $1.1 \times 10^{5}$ & $7.0 \times 10^{22}$ & $\mathrm{~N}$ \\
\hline 23 & 345.443 & 1.387 & 3.5 & 0.4 & $1.2 \times 10^{2}$ & $7.9 \times 10^{4}$ & $6.0 \times 10^{22}$ & $\mathrm{~N}$ \\
\hline 24 & 345.453 & 1.386 & 3.2 & 0.4 & $1.1 \times 10^{2}$ & $7.4 \times 10^{4}$ & $5.5 \times 10^{22}$ & $\mathrm{~N}$ \\
\hline 25 & 345.406 & 1.394 & 2.9 & 0.3 & 95 & $1.3 \times 10^{5}$ & $7.6 \times 10^{22}$ & $\mathrm{Y}$ \\
\hline 26 & 345.389 & 1.531 & 2.0 & 0.3 & 66 & $1.2 \times 10^{5}$ & $6.5 \times 10^{22}$ & $\mathrm{Y}$ \\
\hline 27 & 345.554 & 1.508 & 1.7 & 0.2 & 58 & $1.4 \times 10^{5}$ & $7.0 \times 10^{22}$ & $\mathrm{~N}$ \\
\hline 28 & 345.398 & 1.538 & 1.7 & 0.2 & 55 & $1.4 \times 10^{5}$ & $6.7 \times 10^{22}$ & $\mathrm{~N}$ \\
\hline 29 & 345.244 & 1.021 & 1.8 & 0.3 & 58 & $9.9 \times 10^{4}$ & $5.5 \times 10^{22}$ & $\mathrm{~N}$ \\
\hline 30 & 345.301 & 1.040 & 1.8 & 0.3 & 60 & $1.2 \times 10^{5}$ & $6.3 \times 10^{22}$ & $\mathrm{Y}$ \\
\hline 31 & 345.525 & 1.567 & 1.8 & 0.3 & 59 & $6.4 \times 10^{4}$ & $4.1 \times 10^{22}$ & $\mathrm{~N}$ \\
\hline 32 & 345.396 & 1.389 & 1.5 & - & 49 & - & - & Y \\
\hline 33 & 345.251 & 1.038 & 2.1 & 0.3 & 68 & $1.1 \times 10^{5}$ & $6.3 \times 10^{22}$ & $\mathrm{~N}$ \\
\hline 34 & 344.878 & 1.437 & 3.9 & 0.5 & $1.3 \times 10^{2}$ & $4.1 \times 10^{4}$ & $4.0 \times 10^{22}$ & $\mathrm{Y}$ \\
\hline 35 & 345.353 & 1.448 & 2.5 & 0.5 & 82 & $3.1 \times 10^{4}$ & $2.8 \times 10^{22}$ & $\mathrm{Y}$ \\
\hline 36 & 345.236 & 1.040 & 2.1 & 0.4 & 70 & $5.3 \times 10^{4}$ & $3.9 \times 10^{22}$ & $\mathrm{~N}$ \\
\hline 37 & 344.946 & 1.796 & 1.6 & 0.4 & 54 & $3.7 \times 10^{4}$ & $2.8 \times 10^{22}$ & $\mathrm{~N}$ \\
\hline 38 & 345.442 & 1.558 & 1.2 & 0.2 & 41 & $1.1 \times 10^{5}$ & $5.3 \times 10^{22}$ & $\mathrm{~N}$ \\
\hline 39 & 345.257 & 1.073 & 1.6 & 0.3 & 54 & $7.4 \times 10^{4}$ & $4.4 \times 10^{22}$ & $\mathrm{Y}$ \\
\hline 40 & 345.368 & 1.036 & 1.6 & 0.5 & 53 & $1.9 \times 10^{4}$ & $1.8 \times 10^{22}$ & $\mathrm{Y}$ \\
\hline 41 & 344.942 & 1.241 & 0.55 & - & 18 & - & - & $\mathrm{N}$ \\
\hline 42 & 345.539 & 1.567 & 1.5 & 0.3 & 49 & $4.5 \times 10^{4}$ & $3.1 \times 10^{22}$ & $\mathrm{~N}$ \\
\hline 43 & 345.254 & 1.052 & 1.9 & 0.4 & 64 & $5.2 \times 10^{4}$ & $3.7 \times 10^{22}$ & $\mathrm{~N}$ \\
\hline 44 & 344.904 & 1.803 & 1.9 & 0.4 & 62 & $4.3 \times 10^{4}$ & $3.3 \times 10^{22}$ & $\mathrm{~N}$ \\
\hline 45 & 345.502 & 0.842 & 0.88 & 0.3 & 29 & $4.4 \times 10^{4}$ & $2.6 \times 10^{22}$ & $\mathrm{~N}$ \\
\hline 46 & 345.559 & 1.531 & 0.85 & 0.3 & 28 & $5.2 \times 10^{4}$ & $2.8 \times 10^{22}$ & $\mathrm{~N}$ \\
\hline 47 & 345.360 & 1.389 & 0.80 & 0.2 & 26 & $6.2 \times 10^{4}$ & $3.1 \times 10^{22}$ & Y \\
\hline 48 & 345.441 & 0.206 & 1.8 & 0.5 & 61 & $2.2 \times 10^{4}$ & $2.0 \times 10^{22}$ & $\mathrm{Y}$ \\
\hline 49 & 345.137 & 1.564 & 0.61 & 0.2 & 20 & $5.7 \times 10^{4}$ & $2.7 \times 10^{22}$ & $\mathrm{Y}$ \\
\hline 50 & 345.312 & 1.046 & 0.95 & 0.3 & 31 & $5.0 \times 10^{4}$ & $2.9 \times 10^{22}$ & $\mathrm{Y}$ \\
\hline 51 & 345.445 & 1.371 & 1.3 & 0.5 & 42 & $1.5 \times 10^{4}$ & $1.4 \times 10^{22}$ & $\mathrm{~N}$ \\
\hline 52 & 345.334 & 1.428 & 1.5 & 0.4 & 49 & $3.6 \times 10^{4}$ & $2.6 \times 10^{22}$ & Y \\
\hline 53 & 345.563 & 1.486 & 1.1 & 0.3 & 35 & $6.3 \times 10^{4}$ & $3.5 \times 10^{22}$ & $\mathrm{~N}$ \\
\hline 54 & 344.929 & 1.836 & 0.50 & - & 17 & - & - & $\mathrm{N}$ \\
\hline 55 & 345.335 & 1.434 & 1.1 & 0.5 & 36 & $1.1 \times 10^{4}$ & $1.1 \times 10^{22}$ & $\mathrm{Y}$ \\
\hline 56 & 345.434 & 1.445 & 1.00 & 0.3 & 33 & $7.5 \times 10^{4}$ & $3.8 \times 10^{22}$ & $\mathrm{~N}$ \\
\hline 57 & 345.211 & 1.049 & 0.55 & 0.3 & 18 & $3.7 \times 10^{4}$ & $2.0 \times 10^{22}$ & $\mathrm{~N}$ \\
\hline 58 & 345.968 & 0.598 & 1.5 & 0.4 & 49 & $3.0 \times 10^{4}$ & $2.3 \times 10^{22}$ & $\mathrm{~N}$ \\
\hline 59 & 344.945 & 1.229 & 0.43 & - & 14 & - & - & $\mathrm{N}$ \\
\hline 60 & 345.006 & 1.532 & 1.1 & 0.4 & 36 & $2.5 \times 10^{4}$ & $1.9 \times 10^{22}$ & $\mathrm{Y}$ \\
\hline 61 & 345.265 & 1.085 & 1.2 & 0.3 & 39 & $3.6 \times 10^{4}$ & $2.5 \times 10^{22}$ & $\mathrm{~N}$ \\
\hline 62 & 344.952 & 1.195 & 0.88 & 0.3 & 29 & $6.6 \times 10^{4}$ & $3.3 \times 10^{22}$ & $\mathrm{~N}$ \\
\hline 63 & 345.337 & 1.037 & 0.99 & 0.4 & 33 & $1.7 \times 10^{4}$ & $1.4 \times 10^{22}$ & Y \\
\hline
\end{tabular}


Table 5. continued.

\begin{tabular}{|c|c|c|c|c|c|c|c|c|}
\hline Name & $\begin{array}{l}\text { Galactic } \\
\text { long. }\end{array}$ & $\begin{array}{l}\text { coord. } \\
\text { lat. }\end{array}$ & $\begin{array}{l}S_{1.2 \mathrm{~mm}} \\
\text { Jy }\end{array}$ & $\begin{array}{l}\text { Diameter } \\
\text { pc }\end{array}$ & $\begin{array}{l}\text { Mass } \\
M_{\odot}\end{array}$ & $\begin{array}{l}\text { Density } \\
\mathrm{cm}^{-3}\end{array}$ & $\begin{array}{l}\text { Column density } \\
\mathrm{cm}^{-2}\end{array}$ & $\begin{array}{c}\text { Infrared } \\
\text { counterpart }\end{array}$ \\
\hline 64 & 345.436 & 1.418 & 0.67 & 0.3 & 22 & $4.0 \times 10^{4}$ & $2.2 \times 10^{22}$ & $\mathrm{~N}$ \\
\hline 65 & 345.501 & 0.418 & 0.66 & 0.2 & 22 & $5.4 \times 10^{4}$ & $2.7 \times 10^{22}$ & $\mathrm{~N}$ \\
\hline 66 & 345.457 & 0.428 & 0.81 & 0.3 & 27 & $3.6 \times 10^{4}$ & $2.2 \times 10^{22}$ & $\mathrm{~N}$ \\
\hline 67 & 344.933 & 1.251 & 0.73 & 0.2 & 24 & $7.1 \times 10^{4}$ & $3.3 \times 10^{22}$ & $\mathrm{~N}$ \\
\hline 68 & 345.064 & 1.747 & 0.57 & 0.2 & 19 & $6.0 \times 10^{4}$ & $2.7 \times 10^{22}$ & $\mathrm{~N}$ \\
\hline 69 & 345.501 & 0.389 & 1.3 & 0.5 & 42 & $1.2 \times 10^{4}$ & $1.2 \times 10^{22}$ & $\mathrm{~N}$ \\
\hline 70 & 345.468 & 1.431 & 0.33 & - & 11 & - & - & $\mathrm{Y}$ \\
\hline 71 & 345.558 & 1.522 & 0.40 & - & 13 & - & - & $\mathrm{N}$ \\
\hline 72 & 345.010 & 1.764 & 0.60 & 0.3 & 20 & $1.7 \times 10^{4}$ & $1.2 \times 10^{22}$ & $\mathrm{~N}$ \\
\hline 73 & 345.590 & 1.491 & 0.19 & - & 6.2 & - & - & $\mathrm{N}$ \\
\hline 74 & 344.932 & 1.231 & 0.23 & - & 7.5 & - & - & $\mathrm{N}$ \\
\hline 75 & 344.927 & 1.806 & 0.82 & 0.3 & 27 & $3.0 \times 10^{4}$ & $1.9 \times 10^{22}$ & $\mathrm{~N}$ \\
\hline 76 & 344.947 & 1.813 & 0.48 & 0.4 & 16 & $1.1 \times 10^{4}$ & $8.3 \times 10^{21}$ & $\mathrm{~N}$ \\
\hline 77 & 344.955 & 1.170 & 0.72 & 0.2 & 24 & $6.2 \times 10^{4}$ & $3.0 \times 10^{22}$ & $\mathrm{~N}$ \\
\hline 78 & 344.936 & 1.245 & 0.30 & - & 10 & - & - & $\mathrm{N}$ \\
\hline 79 & 345.384 & 1.038 & 0.68 & 0.3 & 23 & $3.5 \times 10^{4}$ & $2.0 \times 10^{22}$ & $\mathrm{~N}$ \\
\hline 80 & 345.548 & 1.480 & 0.34 & - & 11 & - & - & Y \\
\hline 81 & 345.513 & 0.407 & 0.82 & 0.3 & 27 & $4.6 \times 10^{4}$ & $2.6 \times 10^{22}$ & $\mathrm{~N}$ \\
\hline 82 & 345.563 & 1.526 & 0.36 & 0.2 & 12 & $3.2 \times 10^{4}$ & $1.5 \times 10^{22}$ & $\mathrm{~N}$ \\
\hline 83 & 345.450 & 1.364 & 0.34 & - & 11 & - & - & $\mathrm{N}$ \\
\hline 84 & 345.429 & 1.455 & 0.88 & 0.3 & 29 & $3.7 \times 10^{4}$ & $2.3 \times 10^{22}$ & $\mathrm{~N}$ \\
\hline 85 & 345.010 & 1.617 & 0.45 & - & 15 & - & - & $\mathrm{N}$ \\
\hline 86 & 345.001 & 1.615 & 1.2 & 0.5 & 41 & $1.3 \times 10^{4}$ & $1.3 \times 10^{22}$ & $\mathrm{~N}$ \\
\hline 87 & 345.319 & 1.484 & 0.68 & 0.4 & 22 & $1.8 \times 10^{4}$ & $1.3 \times 10^{22}$ & $\mathrm{Y}$ \\
\hline 88 & 345.519 & 1.639 & 0.71 & 0.3 & 24 & $4.9 \times 10^{4}$ & $2.5 \times 10^{22}$ & $\mathrm{~N}$ \\
\hline 89 & 345.133 & 1.069 & 1.3 & 0.6 & 42 & $7.0 \times 10^{3}$ & $8.4 \times 10^{21}$ & $\mathrm{~N}$ \\
\hline 90 & 345.396 & 1.519 & 0.42 & - & 14 & - & - & Y \\
\hline 91 & 345.033 & 1.632 & 0.76 & 0.3 & 25 & $3.7 \times 10^{4}$ & $2.2 \times 10^{22}$ & $\mathrm{~N}$ \\
\hline 92 & 345.476 & 1.568 & 0.31 & - & 10 & - & - & $\mathrm{N}$ \\
\hline 93 & 344.966 & 1.181 & 0.41 & - & 14 & - & - & $\mathrm{N}$ \\
\hline 94 & 345.240 & 0.390 & 0.49 & - & 16 & - & - & $\mathrm{N}$ \\
\hline 95 & 345.524 & 0.404 & 0.46 & 0.2 & 15 & $3.8 \times 10^{4}$ & $1.9 \times 10^{22}$ & $\mathrm{~N}$ \\
\hline 96 & 345.590 & 0.374 & 0.40 & - & 13 & - & - & $\mathrm{Y}$ \\
\hline 97 & 345.031 & 1.781 & 0.56 & 0.4 & 19 & $9.6 \times 10^{3}$ & $8.0 \times 10^{21}$ & $\mathrm{~N}$ \\
\hline 98 & 345.451 & 0.435 & 0.39 & - & 13 & - & - & $\mathrm{N}$ \\
\hline 99 & 345.078 & 1.786 & 0.64 & 0.3 & 21 & $1.9 \times 10^{4}$ & $1.3 \times 10^{22}$ & $\mathrm{~N}$ \\
\hline 100 & 345.854 & 1.415 & 0.47 & - & 16 & - & - & $\mathrm{N}$ \\
\hline 101 & 345.217 & 0.999 & 0.51 & 0.3 & 17 & $1.8 \times 10^{4}$ & $1.2 \times 10^{22}$ & $\mathrm{~N}$ \\
\hline 102 & 344.936 & 1.596 & 0.44 & - & 15 & - & - & $\mathrm{N}$ \\
\hline 103 & 345.065 & 1.615 & 1.3 & 0.5 & 44 & $1.7 \times 10^{4}$ & $1.5 \times 10^{22}$ & $\mathrm{~N}$ \\
\hline 104 & 345.338 & 1.463 & 0.73 & 0.5 & 24 & $8.8 \times 10^{3}$ & $8.2 \times 10^{21}$ & $\mathrm{~N}$ \\
\hline 105 & 345.585 & 1.484 & 0.41 & 0.2 & 14 & $3.9 \times 10^{4}$ & $1.8 \times 10^{22}$ & $\mathrm{~N}$ \\
\hline 106 & 345.854 & 1.421 & 0.29 & - & 9.5 & - & - & $\mathrm{N}$ \\
\hline 107 & 345.062 & 1.842 & 0.53 & 0.2 & 18 & $5.3 \times 10^{4}$ & $2.4 \times 10^{22}$ & $\mathrm{~N}$ \\
\hline 108 & 345.477 & 1.563 & 0.22 & - & 7.2 & - & - & $\mathrm{N}$ \\
\hline 109 & 345.454 & 1.358 & 0.16 & - & 5.4 & - & - & $\mathrm{N}$ \\
\hline 110 & 345.959 & 0.608 & 0.33 & - & 11 & - & - & $\mathrm{N}$ \\
\hline 111 & 344.949 & 1.213 & 0.27 & - & 8.8 & - & - & $\mathrm{N}$ \\
\hline 112 & 344.942 & 1.589 & 0.45 & - & 15 & - & - & $\mathrm{N}$ \\
\hline 113 & 345.539 & 1.488 & 0.29 & 0.3 & 9.5 & $1.2 \times 10^{4}$ & $7.4 \times 10^{21}$ & $\mathrm{~N}$ \\
\hline 114 & 345.576 & 0.258 & 0.26 & - & 8.5 & - & - & $\mathrm{N}$ \\
\hline 115 & 345.681 & 0.318 & 0.43 & - & 14 & - & - & $\mathrm{N}$ \\
\hline 116 & 345.330 & 1.049 & 0.45 & 0.2 & 15 & $3.9 \times 10^{4}$ & $1.9 \times 10^{22}$ & $\mathrm{~N}$ \\
\hline 117 & 345.776 & 1.445 & 0.34 & - & 11 & - & - & $\mathrm{N}$ \\
\hline 118 & 345.511 & 1.578 & 0.40 & 0.3 & 13 & $1.6 \times 10^{4}$ & $9.8 \times 10^{21}$ & $\mathrm{~N}$ \\
\hline 119 & 344.909 & 1.182 & 0.65 & 0.3 & 22 & $2.6 \times 10^{4}$ & $1.6 \times 10^{22}$ & $\mathrm{~N}$ \\
\hline 120 & 345.518 & 0.835 & 0.34 & - & 11 & - & - & $\mathrm{N}$ \\
\hline 121 & 345.873 & 0.811 & 0.30 & - & 10 & - & - & $\mathrm{N}$ \\
\hline 122 & 345.489 & 0.430 & 0.23 & - & 7.7 & - & - & $\mathrm{N}$ \\
\hline 123 & 344.951 & 1.096 & 0.37 & 0.2 & 12 & $3.7 \times 10^{4}$ & $1.7 \times 10^{22}$ & $\mathrm{~N}$ \\
\hline 124 & 344.906 & 1.196 & 0.38 & 0.2 & 13 & $4.5 \times 10^{4}$ & $2.0 \times 10^{22}$ & $\mathrm{~N}$ \\
\hline 125 & 345.092 & 1.733 & 0.36 & - & 12 & - & - & $\mathrm{N}$ \\
\hline 126 & 345.261 & 1.108 & 0.42 & 0.3 & 14 & $2.1 \times 10^{4}$ & $1.2 \times 10^{22}$ & $\mathrm{~N}$ \\
\hline 127 & 345.467 & 0.419 & 0.45 & 0.2 & 15 & $4.8 \times 10^{4}$ & $2.2 \times 10^{22}$ & $\mathrm{~N}$ \\
\hline
\end{tabular}


A\&A 534, A131 (2011)

Table 5. continued.

\begin{tabular}{|c|c|c|c|c|c|c|c|c|}
\hline Name & $\begin{array}{l}\text { Galactic } \\
\text { long. }\end{array}$ & $\begin{array}{r}\text { oord. } \\
\text { lat. }\end{array}$ & $\begin{array}{c}S_{1.2 \mathrm{~mm}} \\
\mathrm{Jy}\end{array}$ & $\begin{array}{c}\text { Diameter } \\
\text { pc }\end{array}$ & $\begin{array}{c}\text { Mass } \\
M_{\odot} \\
\end{array}$ & $\begin{array}{c}\text { Density } \\
\mathrm{cm}^{-3}\end{array}$ & $\begin{array}{c}\text { Column density } \\
\mathrm{cm}^{-2}\end{array}$ & $\begin{array}{c}\text { Infrared } \\
\text { counterpart }\end{array}$ \\
\hline 128 & 344.801 & 1.082 & 0.27 & - & 8.9 & - & - & $\mathrm{N}$ \\
\hline 129 & 345.482 & 1.233 & 0.23 & - & 7.5 & - & - & $\mathrm{N}$ \\
\hline 130 & 345.464 & 1.443 & 0.21 & - & 7.0 & - & - & Y \\
\hline 131 & 344.849 & 1.312 & 0.24 & - & 7.9 & - & - & $\mathrm{N}$ \\
\hline 132 & 345.484 & 0.856 & 0.17 & - & 5.5 & - & - & $\mathrm{N}$ \\
\hline 133 & 345.304 & 0.435 & 0.12 & - & 3.9 & - & - & $\mathrm{N}$ \\
\hline 134 & 344.945 & 1.600 & 0.64 & 0.3 & 21 & $2.1 \times 10^{4}$ & $1.4 \times 10^{22}$ & $\mathrm{~N}$ \\
\hline 135 & 344.950 & 1.587 & 0.22 & - & 7.3 & - & - & $\mathrm{N}$ \\
\hline 136 & 345.272 & 1.051 & 0.36 & 0.3 & 12 & $1.7 \times 10^{4}$ & $9.8 \times 10^{21}$ & $\mathrm{~N}$ \\
\hline 137 & 345.060 & 1.727 & 0.37 & - & 12 & - & - & $\mathrm{N}$ \\
\hline 138 & 345.479 & 0.400 & 0.26 & - & 8.7 & - & - & $\mathrm{N}$ \\
\hline 139 & 344.846 & 1.293 & 0.21 & - & 7.1 & - & - & $\mathrm{N}$ \\
\hline 140 & 345.909 & 0.514 & 0.36 & 0.2 & 12 & $3.4 \times 10^{4}$ & $1.6 \times 10^{22}$ & $\mathrm{~N}$ \\
\hline 141 & 345.812 & 1.756 & 0.26 & - & 8.5 & - & - & $\mathrm{N}$ \\
\hline 142 & 344.957 & 1.188 & 0.24 & - & 7.9 & - & - & $\mathrm{N}$ \\
\hline 143 & 345.320 & 1.417 & 0.29 & 0.4 & 9.6 & $7.6 \times 10^{3}$ & $5.5 \times 10^{21}$ & Y \\
\hline 144 & 345.869 & 1.820 & 0.21 & - & 6.8 & - & - & $\mathrm{N}$ \\
\hline 145 & 345.278 & 1.112 & 0.35 & 0.2 & 12 & $2.7 \times 10^{4}$ & $1.4 \times 10^{22}$ & $\mathrm{~N}$ \\
\hline 146 & 345.634 & 0.770 & 0.33 & 0.2 & 11 & $3.5 \times 10^{4}$ & $1.6 \times 10^{22}$ & $\mathrm{~N}$ \\
\hline 147 & 345.044 & 1.618 & 0.32 & 0.3 & 11 & $2.4 \times 10^{4}$ & $1.2 \times 10^{22}$ & $\mathrm{~N}$ \\
\hline 148 & 344.809 & 1.075 & 0.12 & - & 3.8 & - & - & $\mathrm{N}$ \\
\hline 149 & 345.683 & 1.459 & 0.30 & 0.3 & 9.9 & $2.2 \times 10^{4}$ & $1.1 \times 10^{22}$ & $\mathrm{~N}$ \\
\hline 150 & 345.566 & 0.267 & 0.17 & - & 5.7 & - & - & $\mathrm{N}$ \\
\hline 151 & 344.925 & 1.316 & 0.33 & - & 11 & - & - & $\mathrm{N}$ \\
\hline 152 & 345.699 & 1.508 & 0.18 & - & 5.9 & - & - & $\mathrm{N}$ \\
\hline 153 & 345.286 & 0.412 & 0.14 & - & 4.6 & - & - & $\mathrm{N}$ \\
\hline 154 & 345.611 & 0.365 & 0.27 & - & 8.8 & - & - & $\mathrm{N}$ \\
\hline 155 & 344.916 & 1.826 & 0.17 & - & 5.5 & - & - & $\mathrm{N}$ \\
\hline 156 & 345.472 & 0.412 & 0.22 & - & 7.2 & - & - & $\mathrm{N}$ \\
\hline 157 & 345.038 & 1.848 & 0.28 & 0.2 & 9.2 & $2.9 \times 10^{4}$ & $1.3 \times 10^{22}$ & $\mathrm{~N}$ \\
\hline 158 & 345.792 & 1.757 & 0.17 & - & 5.7 & - & - & $\mathrm{N}$ \\
\hline 159 & 345.254 & 1.012 & 0.089 & - & 3.0 & - & - & $\mathrm{N}$ \\
\hline 160 & 345.708 & 1.456 & 0.24 & - & 8.0 & - & - & $\mathrm{N}$ \\
\hline 161 & 345.472 & 1.231 & 0.71 & 0.4 & 24 & $1.3 \times 10^{4}$ & $1.1 \times 10^{22}$ & $\mathrm{~N}$ \\
\hline 162 & 345.497 & 1.437 & 0.19 & 0.3 & 6.4 & $8.0 \times 10^{3}$ & $5.0 \times 10^{21}$ & $\mathrm{~N}$ \\
\hline 163 & 344.783 & 1.105 & 0.28 & - & 9.3 & - & - & $\mathrm{N}$ \\
\hline 164 & 345.638 & 0.330 & 0.11 & - & 3.7 & - & - & $\mathrm{N}$ \\
\hline 165 & 345.699 & 1.853 & 0.21 & - & 7.1 & - & - & $\mathrm{N}$ \\
\hline 166 & 344.986 & 1.109 & 0.10 & - & 3.4 & - & - & $\mathrm{N}$ \\
\hline 167 & 345.018 & 1.762 & 0.17 & - & 5.7 & - & - & $\mathrm{Y}$ \\
\hline 168 & 344.893 & 1.335 & 0.45 & 0.4 & 15 & $1.0 \times 10^{4}$ & $7.8 \times 10^{21}$ & $\mathrm{~N}$ \\
\hline 169 & 344.956 & 1.348 & 0.47 & 0.4 & 16 & $6.2 \times 10^{3}$ & $5.6 \times 10^{21}$ & $\mathrm{~N}$ \\
\hline 170 & 345.948 & 0.723 & 0.14 & - & 4.5 & - & - & $\mathrm{N}$ \\
\hline 171 & 344.891 & 1.345 & 0.20 & - & 6.6 & - & - & $\mathrm{N}$ \\
\hline 172 & 344.924 & 1.824 & 0.19 & - & 6.4 & - & - & $\mathrm{N}$ \\
\hline 173 & 344.929 & 1.610 & 0.15 & - & 5.0 & - & - & $\mathrm{N}$ \\
\hline 174 & 345.247 & 0.385 & 0.18 & - & 5.9 & - & - & $\mathrm{N}$ \\
\hline 175 & 345.547 & 0.960 & 0.13 & - & 4.4 & - & - & $\mathrm{N}$ \\
\hline 176 & 344.892 & 1.847 & 0.25 & - & 8.3 & - & - & $\mathrm{N}$ \\
\hline 177 & 345.888 & 1.414 & 0.26 & 0.2 & 8.6 & $2.3 \times 10^{4}$ & $1.1 \times 10^{22}$ & $\mathrm{~N}$ \\
\hline 178 & 345.510 & 0.159 & 0.095 & - & 3.2 & - & - & $\mathrm{N}$ \\
\hline 179 & 345.418 & 1.469 & 0.15 & 0.2 & 4.8 & $1.7 \times 10^{4}$ & $7.5 \times 10^{21}$ & $\mathrm{~N}$ \\
\hline 180 & 344.920 & 1.419 & 0.17 & - & 5.5 & - & - & $\mathrm{N}$ \\
\hline 181 & 345.079 & 1.847 & 0.12 & - & 4.1 & - & - & $\mathrm{N}$ \\
\hline 182 & 345.236 & 1.000 & 0.20 & - & 6.6 & - & - & $\mathrm{N}$ \\
\hline 183 & 345.375 & 1.571 & 0.14 & - & 4.7 & - & - & $\mathrm{N}$ \\
\hline 184 & 345.684 & 1.516 & 0.14 & - & 4.7 & - & - & $\mathrm{N}$ \\
\hline 185 & 345.532 & 1.634 & 0.13 & - & 4.3 & - & - & $\mathrm{N}$ \\
\hline 186 & 345.697 & 1.459 & 0.19 & - & 6.1 & - & - & $\mathrm{N}$ \\
\hline 187 & 345.501 & 1.415 & 0.23 & 0.3 & 7.5 & $1.6 \times 10^{4}$ & $8.2 \times 10^{21}$ & $\mathrm{~N}$ \\
\hline 188 & 345.816 & 1.765 & 0.23 & - & 7.5 & - & - & $\mathrm{N}$ \\
\hline 189 & 345.086 & 1.611 & 0.32 & 0.4 & 11 & $5.2 \times 10^{3}$ & $4.4 \times 10^{21}$ & $\mathrm{~N}$ \\
\hline 190 & 345.043 & 1.838 & 0.21 & 0.3 & 7.0 & $1.4 \times 10^{4}$ & $7.5 \times 10^{21}$ & $\mathrm{~N}$ \\
\hline
\end{tabular}


Table 5. continued.

\begin{tabular}{|c|c|c|c|c|c|c|c|c|}
\hline Name & $\begin{array}{l}\text { Galactic } \\
\text { long. }\end{array}$ & $\begin{array}{r}\text { oord. } \\
\text { lat. }\end{array}$ & $\begin{array}{c}S_{1.2 \mathrm{~mm}} \\
\mathrm{Jy}\end{array}$ & $\begin{array}{c}\text { Diameter } \\
\mathrm{pc}\end{array}$ & $\begin{array}{c}\text { Mass } \\
M_{\odot} \\
\end{array}$ & $\begin{array}{c}\text { Density } \\
\mathrm{cm}^{-3}\end{array}$ & $\begin{array}{c}\text { Column density } \\
\mathrm{cm}^{-2}\end{array}$ & $\begin{array}{c}\text { Infrared } \\
\text { counterpart }\end{array}$ \\
\hline 191 & 345.442 & 1.572 & 0.11 & - & 3.8 & - & - & $\mathrm{N}$ \\
\hline 192 & 345.468 & 1.225 & 0.13 & - & 4.2 & - & - & $\mathrm{N}$ \\
\hline 193 & 344.951 & 1.082 & 0.34 & 0.3 & 11 & $9.6 \times 10^{3}$ & $6.7 \times 10^{21}$ & $\mathrm{~N}$ \\
\hline 194 & 345.482 & 0.431 & 0.27 & 0.3 & 8.8 & $1.9 \times 10^{4}$ & $9.8 \times 10^{21}$ & $\mathrm{~N}$ \\
\hline 195 & 344.913 & 1.165 & 0.13 & - & 4.4 & - & - & $\mathrm{N}$ \\
\hline 196 & 344.933 & 1.820 & 0.17 & - & 5.7 & - & - & $\mathrm{N}$ \\
\hline 197 & 345.458 & 1.559 & 0.13 & 0.2 & 4.5 & $1.1 \times 10^{4}$ & $5.3 \times 10^{21}$ & $\mathrm{~N}$ \\
\hline 198 & 345.792 & 1.440 & 0.096 & - & 3.2 & - & - & $\mathrm{N}$ \\
\hline 199 & 345.098 & 1.726 & 0.11 & - & 3.7 & - & - & $\mathrm{N}$ \\
\hline 200 & 345.293 & 1.093 & 0.14 & - & 4.5 & - & - & $\mathrm{N}$ \\
\hline 201 & 344.991 & 1.150 & 0.11 & - & 3.8 & - & - & $\mathrm{N}$ \\
\hline
\end{tabular}

Notes. Column 1 gives clump names; Cols. 2 and 3, Galactic coordinates of peaks in $1.2 \mathrm{~mm}$ continuum emission; Col. 4, $1.2 \mathrm{~mm}$ flux densities; Col. 5, diameters; Col. 6, masses; Col. 7, densities; Col. 8, column densities; and Col. 9, if clumps are detected in all infrared MSX and SpitzerIRAC bands ("Y") or not ("N"). The densities and the column densities are computed assuming a mean molecular weight of $\mu=2.29$. 